Check for updates

Cite this: Phys. Chem. Chem. Phys., $2019,21,22511$

Received 11th June 2019, Accepted 24th September 2019

DOI: 10.1039/c9cp03306h

rsc.li/pccp

\section{A dithiocarbamate anchoring group as a flexible platform for interface engineering $\dagger$}

\author{
Eric Sauter,$\ddagger^{\mathrm{a}}$ Giulia Nascimbeni, $\ddagger^{\mathrm{b}}$ Daniel Trefz, ${ }^{\mathrm{c}}$ Sabine Ludwigs, \\ Egbert Zojer, ${ }^{* b}$ Florian von Wrochem (D) ${ }^{* d}$ and Michael Zharnikov (D)*a
}

\begin{abstract}
The molecular organization and electronic properties of dithiocarbamate (DTC) anchored self-assembled monolayers (SAMs) linked to $\mathrm{Au}(111)$ substrates are studied by a combination of $\mathrm{X}$-ray photoelectron spectroscopy (XPS), near-edge X-ray absorption fine structure (NEXAFS) spectroscopy, and state-of-the-art density functional theory calculations. For that, several piperidine/piperazine precursors with different architecture and substitution patterns are selected. The presented data show that the DTC anchor provides a useful building block for monomolecular self-assembly on coinage metals with both sulfur atoms bonded to the substrate in a way similar to what is usually observed for the more commonly applied thiolate docking group. The combination of the DTC group with the quite flexible piperidine/piperazine cyclic linkers results in a dense molecular packing with an upright orientation of the terminal moieties. The latter comprise phenyl rings bearing various substituents, which enables tuning the interfacial dipole over a wide range. Simulations on two prototypical DTC-docked SAMs help to better understand the experimental observations and provide insight into the local origin of the SAM-induced shifts in the electrostatic energy. In particular, a comparison of measured and simulated XP spectra reveals the significant contribution of the DTC group to the interfacial dipole.
\end{abstract}

\section{Introduction}

Energy level alignment and injection control at the interfaces between electrodes, organic semiconductors, and buffer layers are highly important issues in the context of modern organic electronics and photovoltaics. A popular approach in this regard is the use of self-assembled monolayers (SAMs), ${ }^{1-16}$ which are densely packed molecular assemblies of typically rod-shaped molecules adsorbed on a substrate in a well-defined upright geometry. Generally, such molecules consist of three major building blocks, viz. a docking group that provides anchoring to the substrate, a tail group comprising the "outer" SAM-ambient interface, and a spacer that separates the docking and tail groups and drives the self-assembly. ${ }^{17,18}$ While all these building blocks can contribute to the joint dipole moment of

\footnotetext{
${ }^{a}$ Applied Physical Chemistry, Heidelberg University, Im Neuenheimer Feld 253, 69120 Heidelberg, Germany. E-mail: Michael.Zharnikov@urz.uni-heidelberg.de

${ }^{b}$ Institute of Solid State Physics, NAWI Graz, Graz University of Technology, Petersgasse 16, 8010 Graz, Austria.E-mail: egbert.zojer@tugraz.at

${ }^{c}$ Chair for Structure and Properties of Polymeric Materials, Institute of Polymer Chemistry (IPOC), University of Stuttgart, Pfaffenwaldring 55, 70569 Stuttgart, Germany

${ }^{d}$ Institute of Materials Science, University of Stuttgart, Heisenbergstr. 3,

70569 Stuttgart, Germany. E-mail: vonwrochem@gmx.de

$\dagger$ Electronic supplementary information (ESI) available. See DOI: 10.1039/ c9cp03306h

₹ E. S. and G. N. contributed equally to the present paper.
}

the molecules constituting the film, it is mostly a dipolar tail group $^{2,3,5,10,19}$ or a polar mid-chain moiety ${ }^{20-24}$ which is selected to specifically adjust the interfacial dipole. In contrast, the docking group is predominantly chosen based on its affinity to a particular substrate serving as the electrode or, in the context of organic photovoltaics, also as the buffer layer. Examples for docking groups comprise phosphonic acids for zinc oxide surfaces ${ }^{10,25}$ (a popular interfacial layer material in organic electronics devices), phosphonic acids and triethoxy- or trichlorsilanes for indium tin oxide $e^{9,25,26}$ (standard transparent electrode material for organic solar cells), and thiolates for coinage metal substrates, ${ }^{7,18,19}$ such as gold and silver (frequently used electrode materials).

Recently, as an alternative to thiolates, the dithiocarbamate (DTC) anchoring group ${ }^{27-32}$ has been suggested as a basis for the functionalization of gold nanoparticles, for molecular electronics, and for the fabrication of dipolar SAMs to be applied in organic electronics. ${ }^{33,34}$ In combination with piperazine and piperidine as an intermediate linker, this group provides a versatile and robust platform for the attachment of different spacer moieties and tail groups. In particular, the piperidine/piperazine linker, forming a bridge between the DTC group and the aromatic substituents, ensures rod-like symmetry of the molecular backbone. ${ }^{33,35}$ This allows a perpendicular orientation of the molecular axis relative to the substrate (a feature not available in earlier aromatic DTC derivatives), ${ }^{27,30,36}$ which is favorable in the context of work function engineering. Consequently, a family of 
monolayers was realized, which delivered work functions of SAMcovered Au electrodes between $4.8 \mathrm{eV}$ and $3.2 \mathrm{eV} .{ }^{33}$ Although the performance of DTC-docked systems in polymer junctions has been demonstrated and a first spectroscopic characterization of the films along with molecule-based calculations has been provided, ${ }^{33}$ the precise structure and molecular orientation of DTC-based SAMs is still largely unexplored. In addition, a deeper understanding of the electronic properties of such SAMs is of fundamental interest in view of the largely unexplored potential of the used unconventional docking group. Of particular relevance in this context is the question which of the contained functional blocks is actually responsible for the shift in the energy landscape that gives rise to the comparably large work function changes.

In the present work, to gain this understanding, we applied advanced spectroscopic tools in combination with state-of-theart quantum mechanical simulations of SAM/Au interfaces. This combination of simulations and experiments, on the one hand, provides insight into the molecular orientation and order of these films, which need to be understood to correlate the molecular properties with the electrostatic potential drop across the metal-organic interface. On the other hand, it affords an in-depth and reliable insight into the atomistic origins of potential shifts and charge rearrangements. Consequently, the primary emphasis of the following discussion is put on the two very basic systems, viz. phenyl-piperidine-DTC (PPd) and phenyl-piperazine-DTC (PPz) SAMs on $\mathrm{Au}(111)$ (Fig. 1). These monolayers represent highly suitable model systems, which were specifically designed for the present experiments and selected for the simulations to address the distinct properties of the piperidine-DTC and piperazine-DTC docking platforms. The terminal phenyl ring of both molecules is a non-polar and well-defined structural unit supporting the formation of ordered SAMs. Convenient in the present context, it also provides a marker for the spectroscopic experiments (see below). The other DTC derivatives investigated in this study are derived from these two basic systems (parent compounds) by a substitution of the phenyl ring in the para position or by its fluorination. The structures of the respective SAMs and the used abbreviations are shown in Fig. 1.

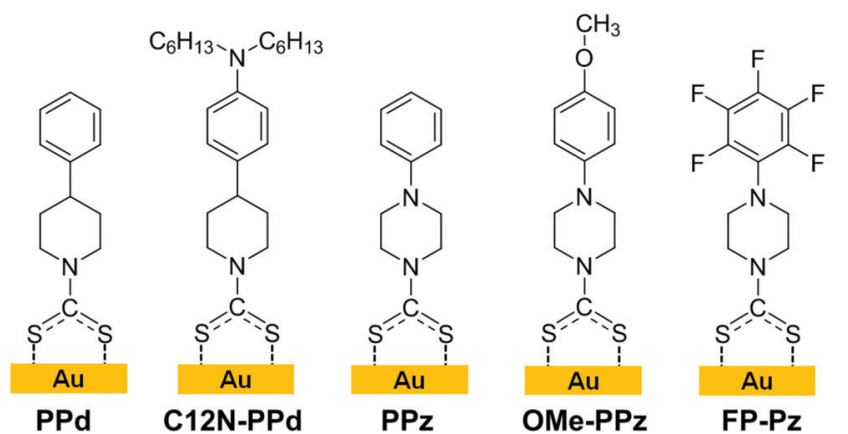

Fig. 1 Schematic structures of the DTC-based SAMs (represented by the single constituents) used in this study along with their respective abbreviations. Note that the C12N-PPd, OMe-PPz, and FP-Pz monolayers have been studied before, but in a somewhat different context compared to the present work. ${ }^{33}$

\section{Experimental and theoretical methods}

\subsection{SAM preparation}

The synthesis of the DTC-based SAM constituents (Fig. 1) was done within an "in situ" process involving coupling the precursor amines to $\mathrm{CS}_{2}$. The amine precursor of C12N-PPd (Fig. 1) was custom-synthesized as reported in the literature. ${ }^{33}$ All other amine precursors for the synthesis of the OMe-PPz, FP-Pz, PPd and PPz DTC derivatives (Fig. 1), i.e. the 1-(4-methoxphenyl)piperazine, 1-(pentafluorophenyl)-piperazine hydrochloride, 4-phenylpiperidine, and 1-phenylpiperazine were obtained in highest purity from ABCR, Sigma Aldrich, Enamine, and Sigma Aldrich, respectively, and used as received. $\mathrm{CS}_{2}$ (p.a.), ethanol (p.a.), and triethylamine were obtained from Sigma Aldrich and Fluka, respectively. The coupling of the precursor amines to $\mathrm{CS}_{2}$ was performed according to the following protocol, established in previous studies. ${ }^{33}$ Initially, concentrated stock solutions of $\mathrm{CS}_{2}(100 \mathrm{mM})$, the amine-terminated precursors $(5 \mathrm{mM})$, and triethylamine $(100 \mathrm{mM})$ were prepared in ethanol. Subsequently, the $\mathrm{CS}_{2}$ and triethylamine solutions, along with some ethanol, were added (in this sequence) to the amine precursor solution to form an equimolar $1: 1: 1$ precursor: $\mathrm{CS}_{2}$ : triethylamine mixture (with a concentration of $1 \mathrm{mM}$ each). The mixture causes a spontaneous reaction of $\mathrm{CS}_{2}$ with the respective amine precursor yielding the desired dithiocarbamate species ( $1 \mathrm{mM})$, which spontaneously and irreversibly reacts with the $\mathrm{Au}(111)$ substrate when the latter is added to the mixture. ${ }^{27,29}$ All steps were carried out in a glove box under nitrogen atmosphere to avoid oxidation reactions. The above DTC solutions were directly used for SAM preparation on gold substrates. As a side note, great care has to be taken in order to avoid any contamination in the glove box, because of the significant risk that thiolate species in the vapor phase might compete with the (rather slow) chemisorption process of dithiocarbamates on $\mathrm{Au}$ (thus compromising SAM purity and uniformity). As substrates, atomically flat template stripped gold (TSG) was employed and prepared according to the reported procedures. ${ }^{37,38}$ The Au films exhibited a root mean square (RMS) roughness of $0.3 \mathrm{~nm}$, exposing preferably (111) oriented surfaces. The organic monolayers were prepared under nitrogen by immersing the freshly stripped TSG substrates into DTC solutions for $\sim 20 \mathrm{~h}$ at room temperature. After immersion, the films were copiously rinsed with ethanol, immediately blown dry with $\mathrm{N}_{2}$ and placed in glass containers, predominantly filled with nitrogen, for transport from the individual laboratories to the synchrotron radiation facilities (see below). No specific degradation was observed on the scale of several days (up to a week) but some minor sample to sample variations were recorded in some of the experiments. All vials and tweezers were cleaned with piranha acid and isopropyl alcohol before usage. In addition, reference SAMs of dodecanethiolate (DDT) and hexadecanethiolate (HDT) were prepared on analogous gold substrates using a standard procedure. ${ }^{39}$

\subsection{SAM characterization}

The fabricated SAMs were characterized by synchrotron-based X-ray photoelectron spectroscopy (XPS) and angle-resolved 
near-edge X-ray absorption fine structure (NEXAFS) spectroscopy. XPS serves as an efficient local probe for the potential distribution within a SAM, at the same time representing a reliable benchmark for theoretical simulations. ${ }^{20,21,40}$ NEXAFS spectroscopy represents a complementary technique, which not only shows high chemical sensitivity but also has the capability of providing information on molecular orientation in adsorbate assemblies and molecular films. ${ }^{41}$ Significantly, such information can be obtained not only for highly ordered systems, but also for samples, in which short- or medium-range order prevails. The latter is frequently the case for SAMs due to structural defects, polymorphism, and a limited size of crystalline domains. These are associated with possible imperfections of the self-assembly process and misfits between the optimal molecular lattice and the structural template provided by the substrate, as well as by the nucleation-type growth of SAMs (leading to domain boundaries). ${ }^{18}$

The measurements were carried out at room temperature under UHV conditions (a base pressure of $\sim 1.5 \times 10^{-9} \mathrm{mbar}$ ). The data acquisition time at a particular sample spot was kept short to minimize possible X-ray induced damage occurring during the measurements. ${ }^{42}$ Experiments were performed at the HE-SGM beamline of the synchrotron storage ring BESSY II (Helmholtz Zentrum Berlin, Germany) and at the D1011 beamline of the MAX IV synchrotron radiation facility (Lund, Sweden). Both are bending magnet beamlines, providing a moderate X-ray intensity, which is a prerequisite for avoiding X-ray induced damage of the comparably sensitive organic films.

XP spectra were acquired using either a Scienta R3000 electron energy analyzer (BESSY II) or a SCIENTA SES200 spectrometer (Max IV) having established performance and reliability. ${ }^{23,24,40,42}$ Spectra acquisition was carried out in normal emission geometry with an energy resolution of either $\sim 0.1 \mathrm{eV}$ (Max IV) or $\sim 0.3 \mathrm{eV}$, $\sim 0.6 \mathrm{eV}$, and $0.7 \mathrm{eV}$ (BESSY II) at excitation energies of $350 \mathrm{eV}$, $580 \mathrm{eV}$, and $720 \mathrm{eV}$, respectively. The binding energy (BE) scale of the XP spectra was referenced to the $A u 4 f_{7 / 2}$ peak of the underlying substrate at a $\mathrm{BE}$ of $84.0 \mathrm{eV}$, at each particular excitation energy. ${ }^{43}$ The obtained spectra were decomposed into individual component peaks and doublets using symmetric Voigt functions and a linear or Shirley-type background. The full width at halfmaximum (fwhm) was kept the same for all individual components within each individual spectrum. To fit the $S 2 p_{3 / 2,1 / 2}$ doublets we used two peaks with the same fwhm, employing the standard $^{43}$ spin-orbit splitting of $\sim 1.18 \mathrm{eV}$ (verified by the fit), and a branching ratio of $2\left(\mathrm{~S} 2 \mathrm{p}_{3 / 2} / \mathrm{S} 2 \mathrm{p}_{1 / 2}\right)$. The accuracy of the resulting $\mathrm{BE}$ and fwhm values is $0.02-0.03 \mathrm{eV}$.

Based on the XPS data, the effective thickness and the packing density of the SAMs were calculated. The evaluation was performed using standard procedures, ${ }^{4,45}$ based on the $\mathrm{C} 1 \mathrm{~s} / \mathrm{Au} 4 \mathrm{f}$ and $\mathrm{S} 2 \mathrm{p} / \mathrm{Au} 4 \mathrm{f}$ intensity ratios. A standard expression for the attenuation of the photoemission signal was assumed (see ESI $\dagger$ and ref. 46) and literature values for the attenuation lengths in densely packed organic films were used, including 11.3 Å for a kinetic energy (KE) of $290 \mathrm{eV}$ (C 1s at a PE of $580 \mathrm{eV}$ ), 15.7 Å for a KE of $490 \mathrm{eV}$ (Au $4 \mathrm{f}$ at a PE of $580 \mathrm{eV}$ ), $8.5 \AA$ for a KE of $185 \mathrm{eV}$ (S 2p at a PE of $350 \mathrm{eV}$ ), and $10.5 \AA$ for a KE of $260 \mathrm{eV}$
(Au 4f at a PE of $350 \mathrm{eV}){ }^{47}$ The spectrometer-specific coefficients were determined using reference monolayers (dodecanethiolate and hexadecanethiolate SAMs on $\mathrm{Au}$ ) of known thickness (14.9 A and 18.9 ̊, respectively) and packing density $\left(4.63 \times 10^{14}\right.$ molecules per $\mathrm{cm}^{2}$ or $21.6 \AA^{2}$ per molecule). ${ }^{39,48}$

The acquisition of the NEXAFS spectra was carried out at the carbon K-edge in the partial electron yield (PEY) mode with a retarding voltage of $-150 \mathrm{~V}$. Linearly polarized synchrotron light with a polarization factor of either $\sim 89 \%$ (Bessy II) or $\sim 95 \%$ (Max IV) was used as the primary X-ray source; the incidence angle was varied to monitor linear dichroism effects. $^{41,49}$ The photon energy (PE) scale was referenced to the pronounced $\pi^{*}$ resonance of highly oriented pyrolytic graphite at $285.38 \mathrm{eV} .{ }^{50}$ Raw NEXAFS spectra were normalized to the incident photon flux determined from the spectrum of a clean, freshly sputtered gold sample. Afterwards, they were reduced to the standard form by subtracting a linear pre-edge background and by normalizing to the unity edge jump.

In addition to the spectroscopic characterization, the work function of the DTC-based SAMs was measured. The measurements were performed using a UHV Kelvin Probe 2001 system (KP technology Ltd, UK). The pressure in the vacuum chamber was $\sim 10^{-9}$ mbar. As a reference, we used a HDT SAM with the work function value set to $4.3 \mathrm{eV}$ according to the literature. ${ }^{22}$ The latter value was additionally verified by its referencing to the work function of freshly sputtered gold set to $5.2 \mathrm{eV} .^{33}$

Note that the work functions measured for the PPz and PPd SAMs displayed some sample to sample variation. Analysis of these data resulted in the values given in Section 3.3; they are considered to be representative of the PPz and PPd monolayers.

\subsection{DFT simulations}

Theoretical simulations were performed for the two most fundamental DTC structures, namely PPd and PPz, as these represent the basic motifs of the investigated class of compounds. A complication in this context is that neither the structure the molecules adopt on the surface, nor the surface unit cell (for the case of commensurate growth) are known. Considering, however, that for PPz essentially the same surface coverage is observed as for the reference SAMs (DDT and HDT; see below), it is reasonable to assume a structure with two molecules in a $(\sqrt{ } 3 \times 3)$ rect unit cell. For the sake of consistency, we chose the same surface unit cell also for the PPd SAM, although there the surface coverage is by $c a$. $10 \%$ lower. The latter could, however, also be caused by a coexistence of differently ordered regions as will be discussed below. The unit cells for the systems with more bulky substituents and even lower surface coverage (C12N-PPd, OMe-PPz, and FP-Pz) are virtually impossible to estimate in the absence of LEED and STM data and we, thus, refrain from modelling those SAMs. With the surface unit cell fixed, the next complication arises with regard to the choice of the relative arrangement of the two molecules within the unit cell, the positions of the $\mathrm{S}$ atoms, and the molecular conformations (planar vs. twisted). To tackle this issue, in an attempt to sample the most important regions of the extended potential energy surface, we calculated a significant 
number of different structures, as described in detail in the ESI. $\dagger$ The properties of the most relevant conformations are discussed below.

For performing the simulations, we used the FHI-aims code ${ }^{51}$ version 150706 , employing the $\mathrm{PBE}$ functional ${ }^{52}$ in combination with the Tkatchenko-Scheffler dispersion correction ${ }^{53}$ in its parametrization for interfaces. ${ }^{54}$ van der Waals interactions between the substrate $\mathrm{Au}$ atoms were turned off. The systems were simulated using periodic boundary conditions and the repeated slab approach, inserting a vacuum region of at least $20 \AA$ in the $z$ direction and including a self-consistently calculated dipole correction ${ }^{55}$ between the slabs. The dimensions of the $(\sqrt{ } 3 \times 3)$ rect unit cell in the $x$ and $y$ directions were defined according to the calculated Au lattice constant (see ESI $\dagger$ ). The substrate surface was described using five layers of Au; the three bottom layers were held fixed in all calculations. A converged $\Gamma$-centered $9 \times 5 \times 1 k$-point grid was applied. Otherwise, the default "tight" settings of FHI-aims were used, which corresponds to the following settings for the basis functions: The $\mathrm{C}$ and $\mathrm{N}$ atoms were described using a "Tier 2" basis consisting of the minimal basis plus one set of basis functions up to an angular momentum of 3 (d-functions) and one set of basis functions up to an angular momentum of 5 (g-functions). The 'Tier 2' basis for $\mathrm{H}$ consists of two sets of basis functions up to $\mathrm{p}$ and $\mathrm{d}$ functions, respectively. The $\mathrm{S}$ atoms were described using the minimal basis plus the full "Tier 1", consisting of one set of basis functions up to an angular momentum of 4 (f-functions), and the d- and g-functions from the "Tier 2" set. The Au atoms were described using a "Tier 1 " basis, consisting of the minimal basis plus one set of basis functions up to an angular momentum of 6 (h-functions). The cutoff potential of all basis functions was set to $4 \AA$ A. Further details of the employed basis functions can be found in the ESI. $\dagger$

The total energy convergence criterion for the self-consistency cycle was set to $10^{-6} \mathrm{eV}$ and the optimizations were performed until the maximum residual force component per atom was below $0.01 \mathrm{eV} \AA^{-1}$.

To understand the impact of chemical $v s$. electrostatic shifts in the XP spectra, reduced coverage systems with nominal coverages of $1 / 16$ and $1 / 36$ were also considered. This dilutes dipoles to a degree that collective electrostatic effects essentially disappear. ${ }^{21}$ Reduced coverage systems were modeled by replicating the optimized full coverage unit cell. In the obtained supercell only one molecule was kept. The geometries of the reduced coverage systems were not separately optimized to prevent the molecule from lying flat on the surface, which would result in a completely changed electronic structure dominated by Pauli pushback effects. ${ }^{56}$ This would not serve the purpose of solely eliminating electrostatic shifts. To limit the computational cost, for the largest unit cells the metal substrate was modeled with only three Au layers, a reduced basis set, and less tight numerical settings. This was done after convergence tests to ensure that it had only a negligible impact on the obtained results (see ESI $\dagger$ ).

The core-level binding energies were simulated within the initial state approach to avoid artefacts arising from a combination of periodic boundary conditions and explicit excitations in each unit cell. ${ }^{21}$ The latter would, for example, occur when performing calculations based on final state approaches ${ }^{57-64}$ in conjunction with upright standing SAMs, in which the excited electron is put into a state at the Fermi level (i.e., into the metal). Due to collective electrostatic effects, ${ }^{65}$ the resulting dipole in every unit cell would lead to a significant artificial shift of core level energies. The magnitude of that shift would scale with the distance of the considered atom in the SAM from the metal substrate. For obtaining the XP spectra, we followed the procedure described by Taucher et al. ${ }^{21}$ In short, the 1 s core level energies for every $\mathrm{C}$ atom were taken from the atom projected density of states output files. The screening of the core hole by the metal substrate was included using an electrostatic image charge model ${ }^{66,67}$

$$
\varepsilon_{\mathrm{Cls}, \mathrm{screened}}=\varepsilon_{\mathrm{Cls}}+14.340 \frac{1}{4 \varepsilon \cdot\left(z-z_{0}\right)}
$$

where $\varepsilon$ is the dielectric constant of the SAM, $z$ is the position of the respective $\mathrm{C}$ atom perpendicular to the surface, and $z_{0}$ is the image plane position (both given in $[\AA]$ ). The energy is obtained in $\mathrm{eV}$ when using the conversion factor of 14.340 (given by the product of the conversion factors between Hartree and $\mathrm{eV}$, respectively, Bohr and $\AA$ ). The position of the image plane was set to $0.9 \AA$ above the average $z$-position of the Au atoms of the topmost layer, consistent with estimates based on comparing image-charge corrections with DFT calculated exchange-correlation potentials for Au surfaces. ${ }^{68,69}$ For the full coverage cases, $\varepsilon$ was set to $2.26,{ }^{70}$ while for the reduced coverage cases it was set to 1.0. Note that this approach takes neither the finite thickness of the SAM nor screening within the dielectric into account. To model the spectra, the obtained (screened) core-level energies were convoluted with Gaussian functions with a variance of $0.19 \mathrm{eV}$ for both PPz and PPd films. The contribution of every atom was weighted using an exponential attenuation function, considering the finite escape depth of the photoelectrons ${ }^{47}$ via an attenuation length set according to a primary photon energy of $350 \mathrm{eV}$ (for more details see ESI $\dagger$ ).

\section{Results and discussion}

\subsection{Experiments: XPS}

The S 2p, C 1s, and N 1s XP spectra of the DTC-based SAMs are presented in Fig. 2 . The $\mathrm{S} 2 \mathrm{p}$ spectra of all films exhibit a single $\mathrm{S} 2 \mathrm{p}_{3 / 2,1 / 2}$ doublet at a BE position of 161.82-161.85 eV (S 2 $\left.\mathrm{p}_{3 / 2}\right)$, as previously shown for dithiocarbamates in general ${ }^{27}$ and for the C12N-PPd, OMe-PPz, and FP-Pz monolayers in particular. ${ }^{33}$ Significantly, the BE of this doublet is lower by $\sim 0.15 \mathrm{eV}$ than the characteristic value for thiolate species bound to noble metal surfaces $(162.0 \pm 0.05 \mathrm{eV}),{ }^{42}$ in agreement with earlier observations. ${ }^{27}$ This suggests a higher negative partial charge on the $\mathrm{S}$ atoms compared to the thiolate sulfur, indeed validated by basic considerations based on DTC resonance hybrid structures (a stronger donating character of the amine group coordinated to the $\mathrm{CS}_{2}$ unit). In this sense, the lower BE of the S 2 p doublet as compared to thiolate does not necessarily mean that each of the sulfur atoms in the DTC moiety is bound stronger to the 


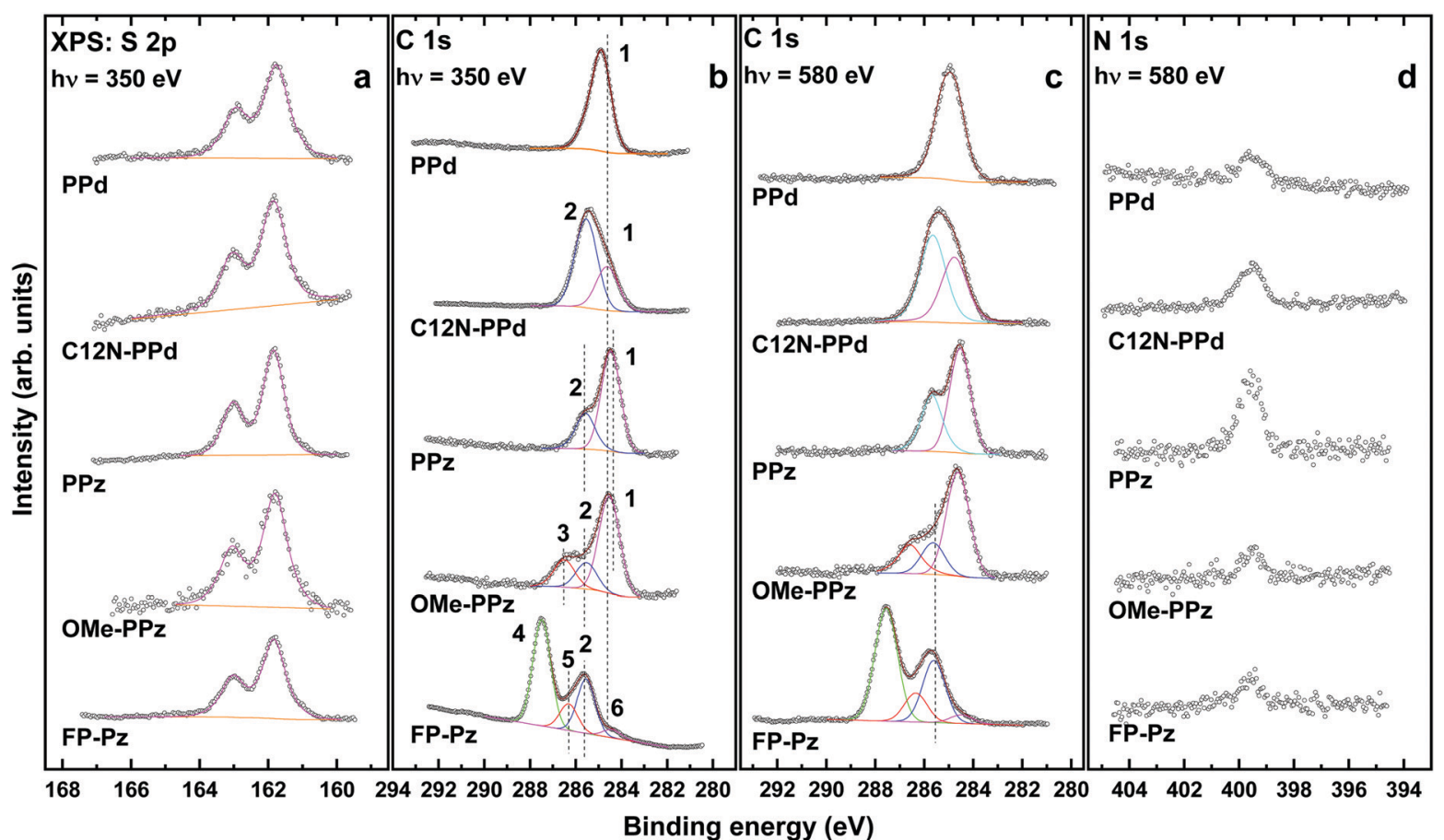

Fig. 2 S 2p (a), C 1s (b and c), and N 1s (d) XP spectra of the DTC-based SAMs. The spectra were acquired at photon energies of 350 eV (S $2 p$ and C 1s in panel b) and $580 \mathrm{eV}$ (C 1s in panel $\mathrm{c}$ and N 1s). The $\mathrm{S} 2 \mathrm{p}$ spectra are fitted by a single $S 2 p_{3 / 2,1 / 2}$ doublet. The $C$ 1s spectra, normalized to the peak height, are decomposed into individual component peaks shown in different colors and marked by numbers, separately for the PPd and PPz related films; see the text for details. The vertical dashed lines are guides to the eyes.

substrate than in the thiolate. This is, however, the case for the entire DTC moiety, as demonstrated by thermal stability experiments in which the desorption peak of $n$-butanethiol assembled on $\mathrm{Au}(111)$ was observed at a noticeably lower temperature $(380 \pm 10 \mathrm{~K})$ than that of a test DTC compound $(470 \pm 10 \mathrm{~K}) .^{27}$ Also, the occurrence of just a single doublet at a BE of $\sim 161.85 \mathrm{eV}$ is significant, as it serves as direct evidence for the formation of SAMs with chemically homogeneous bonding to the substrate. This contrasts positively the situation encountered usually for thiol- and thioether-based ligands with multiple attachment points, where the formation of a variety of different chemical species such as thiolates, atomic sulfur, unbound sulfur, etc. is commonly observed (identified through core level shifts in their $\mathrm{S} 2 \mathrm{p}_{3 / 2}$ signal). ${ }^{71-74}$ The bonding of the DTC-based SAMs to the substrate in a "thiolate-like fashion" also provides a good chemical link. This is an advantage for efficient charge transport, which is important in the context of organic and molecular electronics applications.

The spacing between both $\mathrm{S}$ atoms in the DTC group amounts to $\sim 3.06 \AA$ (calculated employing the methodology described in Section 2.3 both for the isolated, $\mathrm{H}$-saturated molecules and for the densely-packed SAMs). As this value does not fit the periodicity of the $\mathrm{Au}(111)$ template, multiple adsorption sites can be expected. This is indeed implied by the rather high value for the fwhm of the $S 2 \mathrm{p}_{3 / 2,1 / 2}$ components for the DTC-based SAMs, viz. $0.73-0.93 \mathrm{eV}$ compared to the dodecanthiolate monolayers measured under analogous conditions, viz. $0.54-0.59 \mathrm{eV}^{42}$ Consistently, also in the simulations assuming a very high degree of order with two molecules in the unit cell, inequivalent docking positions of the $\mathrm{S}$ atoms were found (see $\mathrm{ESI} \dagger$ ).
Interestingly, the fwhm value for the PPz SAMs $(0.73 \mathrm{eV})$ is somewhat smaller than that for their derivatives, OMe-PPz, and FP-Pz ( 0.84 and $0.80 \mathrm{eV}$, respectively). This suggests an even larger heterogeneity of the adsorption sites (configurations) in the latter cases. The same applies to the PPd and C12N-PPd monolayers, although here the difference $(0.89 \mathrm{eV} v s .0 .93 \mathrm{eV})$ is somewhat smaller.

The C 1s spectra of the DTC-based SAMs in Fig. $2 \mathrm{~b}$ and $\mathrm{c}$ can be decomposed into several component peaks denoted by numbers in the plot. The assignment of these peaks to specific functional groups and specific $\mathrm{C}$ atoms is based on electronegativity considerations, ${ }^{33}$ the comparison to spectra of systems containing similar building blocks, and the analysis of the spectra acquired at different photon energies. The latter is particularly useful considering the stronger contribution of the buried species at a higher excitation energy $(580 \mathrm{eV} v s$. $350 \mathrm{eV}$ ). For the PPz and PPd SAMs these tentative peak assignments will be refined in Section 3.4 building on the atomistic insights provided by the DFT calculations.

The C 1s spectrum of the PPd SAM exhibits only one, nearly symmetric peak at $\sim 284.9 \mathrm{eV}$ (1) originating from a superposition of core-level excitations of the terminal phenyl ring and the piperidine moiety. A signal associated with the DTC carbon, expected at a significantly higher BE (see Section 3.4), is not perceptible because of its strong attenuation. Similarly, the signal of the two $\mathrm{C}$ atoms in the bottom ring bonded to $\mathrm{N}$, which should also appear at a higher BE, is not clearly resolved and is presumably hidden in the high BE tail of the main peak. The joint contribution of the phenyl ring and piperidine can 
also be identified upon decomposition of the asymmetric peak in the C 1s spectra of the C12N-PPd SAM into two component peaks. The low BE component peak at $\sim 284.7 \mathrm{eV}(\mathbf{1})$ is then assigned to the same $\mathrm{C}$ atoms as the sole peak in PPd, while the high $\mathrm{BE}$ component at $285.4 \mathrm{eV}$ (2) is ascribed to the terminal aliphatic chains. Such a comparably high value for the aliphatic chains is presumably related to electrostatic effects, since similar and even higher values were observed for the alkanethiolate SAMs with embedded dipolar groups. ${ }^{22}$ The intensity of this peak is comparably high since the signal from the phenyl ring and piperidine is more strongly attenuated. This is also the case for the signal stemming from the $\mathrm{C}$ atoms bound to the uppermost $\mathrm{N}$ atom.

The spectra of the PPz SAM exhibit two peaks at $285.5 \mathrm{eV}$ (2) and $284.5 \mathrm{eV}$ (1), according to the fits. They are assigned to the $\mathrm{C}$ atoms bonded to $\mathrm{N}$ atoms (all atoms in the piperazine ring and the lowest atom in the phenyl ring) (2) and to all other $\mathrm{C}$ atoms in the terminal phenyl ring (1), respectively. The origin of the splitting of the peaks in this system will become clear from the discussion in Section 3.4. The above two components can also be identified in the spectra of the OMe-PPz film, where they are accompanied by an additional peak at $286.5 \mathrm{eV}(3)$, which originates from the carbon atom in the terminal $\mathrm{OCH}_{3}$ group. Finally, the piperazine component peak (2) is clearly resolved also in the spectra of the FP-Pz monolayer. The dominant component in these spectra is, however, the peak at $287.5 \mathrm{eV}(\mathbf{4})$, which is related to the fluorine-bonded carbon atoms in the terminal ring. In addition, a low intensity component peak at $286.3 \mathrm{eV}$ (5) can be identified upon spectral decomposition. This peak can be tentatively associated with the carbon atom of the upper ring, which is directly bonded to the piperazine, but is also somewhat affected by the $\mathrm{F}$ atoms in the vicinity. Finally, there is a low BE shoulder at $284.3 \mathrm{eV}(6)$, which cannot be assigned to any functional group of FP-Pz and, most likely, stems from a contamination. This contamination stems most likely from a non-complete self-cleaning upon SAM formation, as can be expected for such a bulky molecule as FP-Pz.

The N 1s spectra of all DTC-based SAMs in Fig. 2d exhibit a single peak at $\sim 399.5 \mathrm{eV}$ assigned to the nitrogen atoms in the piperazine and piperidine rings as well as to the nitrogen atom in the terminal dihexylamine group of the C12N-PPD film. The relative intensities of the $\mathrm{N}$ 1s peaks correlate coarsely with the amount of the nitrogen atoms and their locations within the molecular backbone (affecting the attenuation of the respective signal). The F 1s spectrum of the FP-Pz monolayer (not shown) acquired at a photon energy of $720 \mathrm{eV}$ exhibits a single peak at $\sim 687.9 \mathrm{eV}$, assigned to the fluorine atoms in the terminal ring.
The quantitative evaluation of the XP spectra (see Section 2) yields the values for the effective thicknesses and packing densities of the DTC-based SAMs shown in Table 1. The effective thicknesses of the monolayers are close to the sum of the respective molecular lengths and the length of the S-Au bond, suggesting an upright molecular orientation. The packing densities of the parent PPd and PPz films are very close to the value characteristic of the densely packed $(2 \sqrt{3} \times \sqrt{ } 3) R 30^{\circ}$ structure typical of non-substituted alkanethiolate SAMs on $\mathrm{Au}(111)$, viz. $4.63 \times 10^{14} \mathrm{~mol} \mathrm{~cm}{ }^{-2} \cdot{ }^{39,48}$ In view of the almost double density of the sulfur atoms in the DTC case, the similarity of the packing densities is a direct indication that the packing is mostly determined by the interaction between the molecular backbones rather than by the docking groups. This also implies that the docking groups in conventional thiolate SAMs, with only one $\mathrm{S}$ atom per molecular chain, are comparably loosely packed. An important implication of this finding is that the presence of atomic sulfur, which is frequently observed in thiolate SAMs as a minor contamination, ${ }^{42}$ does not necessarily indicate a poor or limited quality of the monolayers, since on the surface there is, apparently, enough space for the coexistence of thiolate anchors packed in the standard fashion and additional sulfur atoms, without resulting in serious interference.

The packing densities of the C12N-PPd and OMe-PPz SAMs are somewhat lower than those of the parent $\mathrm{PPd}$ and $\mathrm{PPz}$ monolayers (Table 1). For the FP-Pz monolayer a further reduction in the packing density is observed, which can be associated with the much larger van-der-Waals radii of the fluorine atoms compared to hydrogens and the resulting larger van-der-Waals dimension of the fluorinated phenyl. ${ }^{45,75}$ For the C12N-PPd and OMe-PPz SAMs, the lower packing densities are associated with the substituents, which, especially for $\mathrm{C} 12 \mathrm{~N}$ PPd (two aliphatic chains per molecule), are comparably bulky. Moreover, the larger monolayer unit cells resulting from the more extended molecular backbones are presumably also no longer commensurate with the positions of the docking sites on the Au surface. This, together with the flexibility of some of the substituents, is expected to cause an increase of the disorder in the films. An additional factor for OMe-PPz SAMs could be interactions between the polar tail groups, disturbing the molecular packing and leading to a reduction of the packing density. ${ }^{76}$ A further fingerprint of the comparably lower quality of the C12N-PPd and OMe-PPz SAMs is the presence of an additional doublet in the $S 2 p$ spectra of these monolayers (Fig. 2a), appearing as a weak shoulder at the low BE side of the main feature. This doublet, at $\sim 161.0 \mathrm{eV}\left(\mathrm{S} 2 \mathrm{p}_{3 / 2}\right)$, can be either ascribed to atomic sulfur or to a distinctly different

Table 1 Effective thickness $(\AA)$, packing density (molecules per $\mathrm{cm}^{2}$ ), and relative density of the DTC-based SAMs. See the text for details. The experimental errors are $\pm 1-1.5 \AA$ for the thickness and $\pm 10 \%$ for the packing density. The reference packing density for a dodecanethiol SAM ( $\sqrt{ } 3 \times$ $\sqrt{ } 3) R 30^{\circ}$ structure is $4.63 \times 10^{14}$ molecules per $\mathrm{cm}^{2}$

\begin{tabular}{lllll}
\hline Monolayer & PPd & C12N-PPd & PPz & OMe-PPz \\
\hline Thickness $(\AA)$ FP-Pz & 9.9 & 13.3 & 10.8 & 11.9 \\
Packing density $\left(\right.$ molecules per $\left.\mathrm{cm}^{2}\right)$ & $4.25 \times 10^{14}$ & $3.5 \times 10^{14}$ & $4.65 \times 10^{14}$ & $3.4 \times 10^{14}$ \\
Relative density & 0.92 & 0.76 & 1.0 & 0.74
\end{tabular}


(compared to the standard ones) bonding configuration of the $\mathrm{S}$ atom (see discussion in ref. 42 and 77). The atomic sulfur scenario is more realistic in our opinion.

\subsection{Experiments: NEXAFS spectroscopy}

The C K-edge NEXAFS spectra of the DTC-based SAMs acquired at an X-ray incident angle of $55^{\circ}$ (magic angle) are presented in Fig. 3a; the differences between the spectra collected under normal $\left(90^{\circ}\right)$ and grazing $\left(20^{\circ}\right)$ incidence are shown in Fig. $3 \mathrm{~b}$. The $55^{\circ}$ spectra are not affected by molecular orientation effects and are, thus, directly representative of the electronic structure of the studied films. ${ }^{41}$ In contrast, the difference spectra provide insight into the dependence of the magnitude of the absorption resonances on the incidence angle of the primary $\mathrm{X}$-ray beam, termed as linear dichroism. This provides information on preferential molecular orientation. ${ }^{41}$

The $55^{\circ}$ spectra of the basic PPd and PPz SAMs are dominated by the characteristic absorption resonances of the terminal phenyl ring, ${ }^{41,78-80}$ viz. the most intense $\pi_{1}{ }^{*}$ resonance at $\sim 285.1 \mathrm{eV}(1)$, the respective $\pi_{2}{ }^{*}$ peak at $288.8-288.9 \mathrm{eV}(3)$, a further $\pi^{*}$ resonance at $290.2 \mathrm{eV}(4)$, and several $\sigma^{*}$ resonances (5-6) at higher excitation energies. In addition, there are the $\mathrm{R}^{*} / \mathrm{C}-\mathrm{S}^{*}$ resonances $^{41,80}$ at $\sim 287.3 \mathrm{eV}(2)$ and the comparably strong $\pi^{*}$ resonance at $\sim 286.5 \mathrm{eV}(7)$ in the case of the PPz SAM, which stems presumably from the splitting of the $\pi_{1}{ }^{*}$ feature of the phenyl ring (1) due to the effect of the adjacent piperazine moiety. ${ }^{41}$

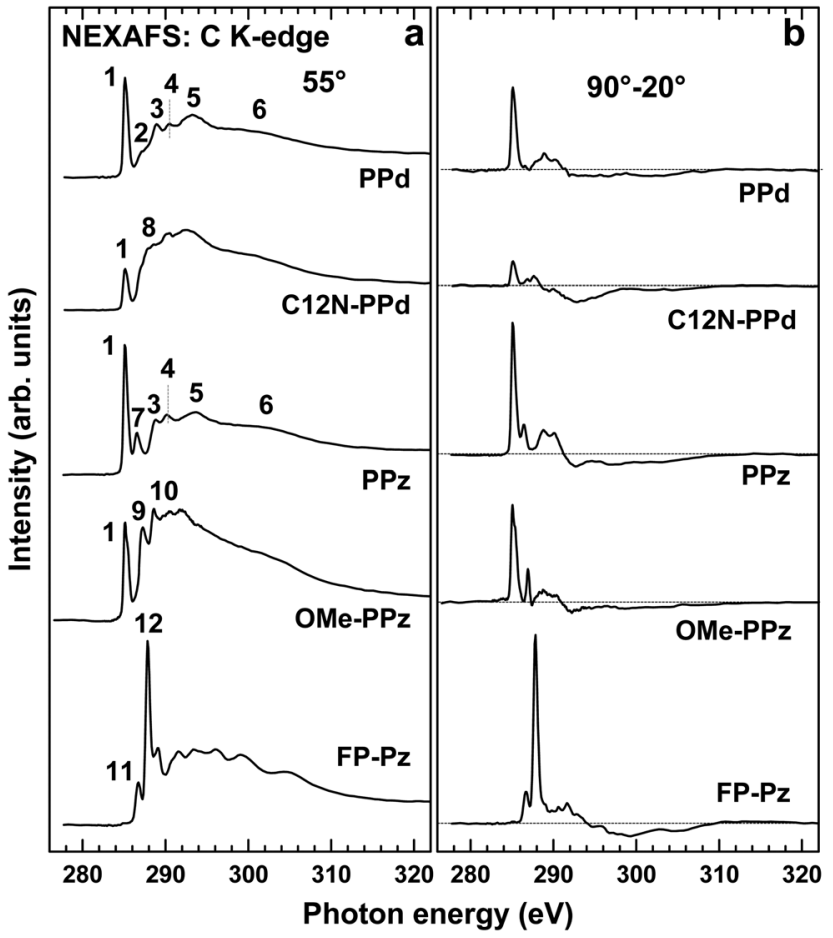

Fig. 3 C K-edge NEXAFS spectra of the DTC-based SAMs acquired at an $X$-ray incident angle of $55^{\circ}$ (a), along with the respective difference between the spectra collected under the normal $\left(90^{\circ}\right)$ and grazing $\left(20^{\circ}\right)$ incidence geometry (b). The same intensity scale is used in both panels. Individual absorption resonances are marked by numbers (see the text for assignments). The horizontal dashed lines in panel b correspond to zero.
Such a splitting also affects the $\pi_{2}{ }^{*}$ resonance (3) in this spectrum, ${ }^{41}$ resulting in a stronger intensity of the $290.2 \mathrm{eV}$ feature (4) compared to the PPd case. The absorption structure associated with the piperidine (in PPd) and piperazine (in PPz) moieties cannot be unequivocally identified, since the spectra of these species do not exhibit intense, discrete features in the pre-edge and at-edge regions (see ref. 81 and 82) and the related $\sigma^{*}$ resonances overlap with those of the phenyl ring.

The $55^{\circ}$ spectra of the C12N-PPd and OMe-PPz SAMs exhibit a significant relative weakening of the $\pi_{1}{ }^{*}$ resonance $(\mathbf{1})$, which is associated with the substitution. This effect is especially pronounced for the C12N-PPd film, the spectrum of which also shows an additional resonance at $\sim 287.7 \mathrm{eV}(8)$ associated with the alkyl chains (most likely excitations into Rydberg states, ${ }^{83}$ but there are also alternative assignments). Additionally, there is a pattern of $\sigma^{*}$ resonances at higher photon energies. The latter resonances stem predominantly from the alkyl chains and are presumably the reason for the comparably low intensity of the $\pi_{1}^{*}$ resonance, since the spectrum is normalized to the height of the absorption edge proportional to the entire number of the carbon atoms. Moreover, we expect a stronger contribution from the alkyl chains due to the more pronounced attenuation of the signal originating from the PPd moiety. The spectrum of the OMe-PPz SAM exhibits a $\pi^{*}$ resonance at $287.3 \mathrm{eV}$ (9) stemming from the splitting of the $\pi_{1}^{*}$ feature of the phenyl ring (1) due to its substitution, ${ }^{41}$ and an additional $\pi^{*}$ resonance at $\sim 288.6 \mathrm{eV}(10)$, which most likely has $\pi_{2}{ }^{*}(3)$ character.

The $55^{\circ}$ spectrum of the FP-Pz SAM is dominated by the absorption resonances of the fluorinated phenyl ring, with the most prominent characteristic features at $\sim 286.65 \mathrm{eV}(\mathbf{1 1})$ and $\sim 287.8 \mathrm{eV}(12)$ assigned to the $\mathrm{C}_{1 \mathrm{~s}} \rightarrow \pi^{*}$ transitions at the carbon atoms which are not bonded and directly bonded to fluorine atoms, respectively. ${ }^{78,84}$ Interestingly, the position of the former feature is distinctly higher than that for pentafluorobenzene $(\sim 285.5 \mathrm{eV})^{78}$ and pentafluorobenzene terminated SAMs $(285.7 \mathrm{eV}) .{ }^{45}$ This is presumably related to the bonding of the respective carbon atom to the adjacent piperazine ring. There are also several additional less intense $\pi^{*}$ - and $\sigma^{*}$-like resonances, for which the positions and assignments can be found in the literature. ${ }^{78,84}$

In addition to the magic angle spectra analyzed above, the linear dichroism effects in the DTC-based SAMs were monitored. The $90^{\circ}-20^{\circ}$ curves for all monolayers in Fig. $3 \mathrm{~b}$ exhibit intense difference peaks at the positions of the absorption resonances. They reflect a pronounced linear dichroism suggesting high orientational order in the monolayers. In addition, the signs of the observed difference peaks, viz. positive signs for resonances with $\pi^{*}$-character and negative signs for those with $\sigma^{*}$-character indicate (in view of the orientation of the respective orbitals) an upright molecular orientation in all studied SAMs.

Along with these qualitative considerations, a quantitative analysis of the entire set of the NEXAFS data acquired at different X-ray incidence angles was performed to get information about molecular orientation in the DTC SAMs. The analysis was carried out within a standard theoretical framework, ${ }^{41}$ comparing the angular dependence of the intensities of selected 
absorption resonances, $I$, with the appropriate theoretical expression. The average angle describing the orientation of the respective molecular orbital in terms of its transition dipole moment (TDM) is used as a fitting parameter. ${ }^{45,80}$ For this analysis we chose either the most prominent resonance associated with the phenyl ring (1) or the most prominent $\pi^{*}$ resonance of the fluorinated phenyl ring $\left(\mathrm{C}_{1 \mathrm{~s}}{ }^{\mathrm{C}-\mathrm{F}} \rightarrow \pi^{*} ; \mathbf{1 2}\right)$, using these moieties as markers for orientation determination. The average tilt angle, $\alpha$, of the corresponding orbitals (with their "direction" defined perpendicular to the plane of the ring) relative to the surface normal can be derived from a standard expression for a vector-type orbital ${ }^{41}$

$$
\begin{aligned}
I(\alpha, \theta)= & A\left\{P \times \frac{1}{3}\left[1+\frac{1}{2} \cdot\left(3 \cdot \cos ^{2} \theta-1\right) \cdot\left(3 \cdot \cos ^{2} \alpha-1\right)\right]\right. \\
& \left.+(1-P) \frac{1}{2} \sin ^{2} \alpha\right\}
\end{aligned}
$$

where $A$ is a constant, $P$ is the polarization degree of the X-rays, and $\theta$ is the X-ray incidence angle. The derived $\alpha$ values are shown in Table 2. They can be used to calculate the average tilt angle of the molecular backbone, $\beta$, according to the relation

$$
\cos (\alpha)=\sin (\beta) \cos (\gamma)
$$

where $\gamma$ is the twist angle of the molecular backbone with respect to the plane spanned by the surface normal and the molecular axis; it is defined as 0 , if the tilt occurs perpendicular to the plane of the ring (see schematic drawings in ref. 24 or 85). In that case, $\beta^{\prime}=90^{\circ}-\alpha$ represents the minimum possible molecular inclination consistent with the NEXAFS data. The respective values are given in Table 2 .

Larger values of the twist angle will result in larger values of the molecular tilt angles for a given $\alpha$. Regretfully, the twist angle cannot be determined experimentally, which is a general constraint of NEXAFS experiments on aromatic SAMs. Therefore, for the most basic PPd and PPz SAMs, we rely on theoretical simulations (Section 3.4), deriving $\beta$ and $\gamma$ from the optimized molecular structures, calculating the respective $\alpha$, and comparing them with the experimental values (such an approach was recently implemented in ref. 24).

Interestingly, the inclination of the $\pi$-system expressed by $\alpha$ in $\mathrm{PPz} / \mathrm{Au}$ is smaller than that in $\mathrm{PPd} / \mathrm{Au}$, in full agreement with the packing density values (Table 1 ). The substitution of the terminal phenyl rings in $\mathrm{C} 12-\mathrm{PPd} / \mathrm{Au}$ and $\mathrm{OMe}-\mathrm{PPz} / \mathrm{Au}$ results in an increase of the inclination, which is a common behavior observed in different types of SAMs. Finally, the fluorination of the terminal phenyl ring in FP-Pz/Au does not result in a

Table 2 Average tilt angle of the $\pi^{\star}$ orbitals of the phenyl ring and the minimal possible average molecular tilt angle for the DTC-based SAMs on gold. See the text for details. The experimental error is estimated at $\pm 3^{\circ}$

\begin{tabular}{llllll}
\hline \multirow{2}{*}{ Monolayer } & \multicolumn{3}{c}{ C12N- } & \multicolumn{2}{c}{ OMe- } \\
\hline Tilt angle of the $\pi^{*}$ orbitals, $\alpha$ & $72.5^{\circ}$ & $70^{\circ}$ & $81.5^{\circ}$ & $72^{\circ}$ & $79^{\circ}$ \\
Minimum molecular tilt angle, $\beta^{\prime}$ & $17.5^{\circ}$ & $20.0^{\circ}$ & $8.5^{\circ}$ & $18.0^{\circ}$ & $11.0^{\circ}$
\end{tabular}

noticeable disturbance of the molecular inclination, which again appears to be a general trend for SAMs containing perfluorinated aromatic moieties. ${ }^{45,86}$

\subsection{Experiments: work function}

The work function values of Au surfaces modified with the PPz and PPd SAMs were determined to be $3.9 \mathrm{eV}$ and $3.7 \mathrm{eV}( \pm 0.05)$, respectively; accordingly, compared to a work function of a reference Au substrate of $5.2 \mathrm{eV}$, the shifts amount to $-1.3 \mathrm{eV}$ and $-1.5 \mathrm{eV}$. The work function values of the C12N-PPd, OMe$\mathrm{PPz}$, and FP-Pz SAMs are $3.2 \mathrm{eV}, 3.5 \mathrm{eV}$, and $4.3 \mathrm{eV}$ according to the literature. ${ }^{33}$ They show the expected trends when compared to the work function values of the parent PPz and PPd derivatives, viz. a decrease in the work function upon substitution with electrondonating groups (C12N and $\mathrm{OMe}$ ) and an increase in the work function upon partial or complete substitution of the terminal moiety with electronegative fluorine atoms (FP-Pz). ${ }^{7,10,19,22}$

\subsection{Quantum-mechanical simulations}

3.4.1. Monolayer structure. In view of the unknown structure of the adsorbate layer, we performed a (restricted) screening of the potential-energy surface of the interface to identify different polymorphs (for details see Section 2.3 and $\mathrm{ESI} \dagger$ ). The relative stabilities and selected properties of the thus-identified structures are shown in Table 3 . The structures of the most stable conformations are shown in Fig. 4 and those of the others are provided in the ESI. $\dagger$ The packing density for the simulated systems with two molecules in a $(\sqrt{ } 3 \times 3)$ rect surface unit cell amounts to $4.45 \times 10^{14} \mathrm{~mol} \mathrm{~cm}{ }^{-2}$, which is equivalent to the experimental packing density of the PPz SAM (see Table 1).

The data in Table 3 reveal that in the SAMs there are different polymorphs that are reasonably close in energy. Interestingly, the lowest energy structures of the two molecules in the SAMs are fundamentally different, with a coplanar structure being most stable for PPz, while for PPd a twisted conformation represents the energetic minimum. This difference is attributed to the

Table 3 DFT-calculated properties of various polymorphs of the PPz/ $\mathrm{Au}(111)$ and PPd/Au(111) interfaces: $\Delta E_{\text {tot }}(\mathrm{eV})$ denotes the total energy per molecule relative to the most stable conformation; the $\alpha$ 's are the average tilt angles of the $\pi$-orbitals (cf., Section 3.2) of the two inequivalent molecules in the unit cell (the individual angles are reported in the ESI). $B E_{C 1 s}$ refers to the energetic position of the main peak in the simulated core-level spectra (rigidly shifted by $18.95 \mathrm{eV}$, to align the simulated and measured spectra of the PPz SAM - for more details see main text); and $\Delta \Phi$ is the SAM-induced work-function change (as reference energy the theoretical work function of the clean $\mathrm{Au}(111)$ surface was used, calculated to be $5.12 \mathrm{eV}$ ). Values for the lowest-energy structures are plotted in bold

\begin{tabular}{llllll}
\hline \multicolumn{2}{c}{ Arrangement } & $\Delta E_{\text {tot }}(\mathrm{eV})$ & $\alpha /^{\circ}$ & $\mathrm{BE}_{\mathrm{C} 1 \mathrm{~s}} / \mathrm{eV}$ & $\Delta \Phi(\mathrm{eV})$ \\
\hline $\mathrm{PPz}$ & Herringbone, coplanar & $\mathbf{0}$ & $\mathbf{8 1 . 2}$ & $\mathbf{2 8 4 . 4 5}$ & $-\mathbf{1 . 7 1}$ \\
& Herringbone, twisted & $0.135^{* *}$ & 83.6 & 285.34 & -2.60 \\
& Cofacial, coplanar & 0.211 & 82.0 & 284.29 & -1.57 \\
& Cofacial, twisted & 0.340 & 89.1 & 285.12 & -2.38 \\
$\mathrm{PPd}$ & & & & \\
& Herringbone, coplanar & 0.083 & 81.2 & 285.26 & -2.54 \\
& Herringbone, twisted & $\mathbf{0}$ & $\mathbf{8 1 . 5}$ & $\mathbf{2 8 5 . 3 0}$ & $-\mathbf{2 . 5 8}$ \\
& Cofacial, coplanar & 0.275 & 73.0 & 285.03 & -2.29 \\
& Cofacial, twisted & 0.214 & 85.5 & 284.98 & -2.29
\end{tabular}



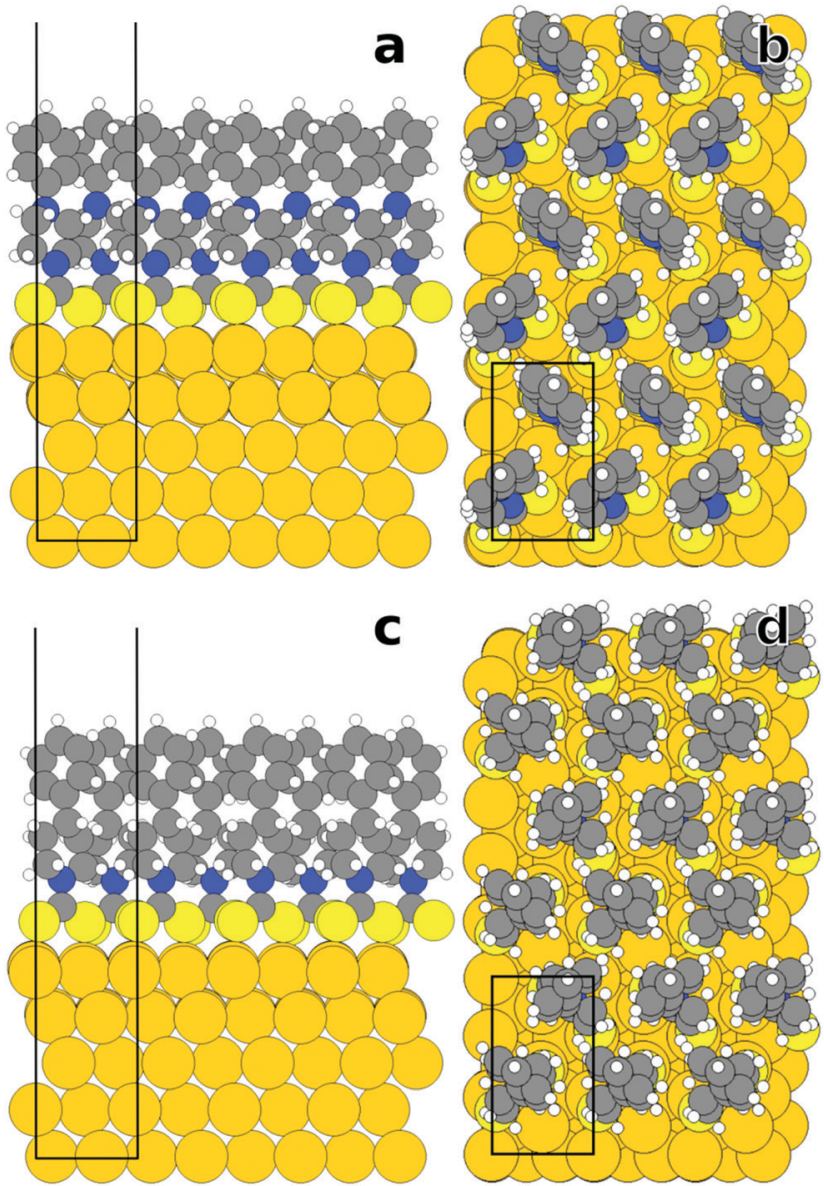

Fig. 4 Optimized structures of PPz/Au(111) and PPd/Au(111). (a) and (b): side and top views of herringbone coplanar PPz/Au(111); (c) and (d): side and top views of herringbone twisted PPd/Au(111). Au atoms are depicted in dark yellow, $\mathrm{S}$ in yellow, $\mathrm{C}$ in grey, $\mathrm{H}$ in white, and $\mathrm{N}$ in blue. The black rectangle marks the unit cell used in the calculations.

kink in the molecular backbone induced by the $\mathrm{sp}^{3}$ hybridized top $\mathrm{C}$ atom in the piperidine ring. This interpretation is supported by the finding that the relative energetic order of twisted/coplanar conformations obtained for the periodic SAMs on the surface is recovered also when calculating isolated molecules. Generally, in all cases two molecules per unit cell arranged in a herringbone pattern are more stable than a cofacial arrangement, consistent with what is known for oligophenylenethiol SAMs. ${ }^{87,88}$ Especially for SAMs containing aromatic entities this packing can be traced back to multipole interactions between the negative charge in the $\pi$-system and the partially positive hydrogen atoms, as explained in detail for biphenylthiolates in ref 89.

The average angle of the $\pi^{*}$ orbitals of the terminal rings relative to the substrate surface (the values of $\alpha$ from Section 3.2) has been obtained via $\cos ^{2}(\alpha)=0.5 \cdot\left(\cos ^{2}\left(\alpha_{1}\right)+\cos ^{2}\left(\alpha_{2}\right)\right)$ from the angles of the individual molecules, $\alpha_{1}$ and $\alpha_{2}$. This takes into consideration that the intensities associated with the absorption resonances are proportional to $\cos ^{2}(\alpha)$ (see eqn (2); the $\sin ^{2}(\alpha)$ component in that equation is comparably small). For the $\mathrm{PPz}$ SAM, we see a near perfect agreement between simulations $\left(81.2^{\circ}\right)$ and experiments $\left(81.5^{\circ}\right)$ when considering the lowest-energy
Table 4 Calculated tilt and twist angles of the two inequivalent molecules in the unit cells of PPz (herringbone, coplanar) and PPd (herringbone, twisted) SAMs at full coverage

\begin{tabular}{lccccc}
\hline & Tilt & & & \multicolumn{2}{c}{ Twist } \\
\cline { 2 - 3 } & $\beta_{1}$ & $\beta_{2}$ & & $\gamma_{1}$ & $\gamma_{2}$ \\
\hline PPz & 7.4 & 13.7 & 37.6 & 36.1 \\
PPd & 14.2 & 12.6 & & 58.0 & 41.1 \\
\hline
\end{tabular}

structure. For the PPd system, the calculated value $\left(81.5^{\circ}\right)$ is larger than the experimental one $\left(72.5^{\circ}\right)$. This deviation is attributed to a non-negligible amount of disorder in the experimentally studied PPd film, which is consistent with the reduced coverage measured for the PPd SAM compared to the PPz monolayer (Table 1). Considering the twisted molecular backbone of PPd in the lowest-energy structure, a reduced film quality for that system appears indeed plausible.

An advantage of the simulations is that they provide direct access to the tilt and twist angles of the individual molecules, which are shown, for the lowest-energy conformations, in Table 4. These data suggest nearly upright-standing molecules in full agreement with the XPS data (see Section 3.1). Notably, the tilt angles calculated for PPd are smaller than the minimum tilt angles derived from the measurements (see Table 2), which can again be attributed to some disorder in the experimentally investigated PPd films.

3.4.2. Calculated core-level shifts and variations in the electrostatic energy. A simulation of the C 1s XP spectra can provide valuable information regarding SAM-induced modifications of the electrostatic potential. ${ }^{20,21}$ In the calculations, we observe a shift of $0.85 \mathrm{eV}$ between the main peaks of the core-level spectra of PPz and PPd. This agrees qualitatively with the experiments, although in absolute numbers the calculated shift between the two systems is clearly larger than the value of $0.42 \mathrm{eV}$ measured at $350 \mathrm{eV}$ photon energy. This difference can at least partly be attributed to the lower effective coverage in the experimental PPd samples, which, on the one hand, dilutes the dipoles and, on the other hand, results in a somewhat larger tilt of the dipole direction relative to the surface normal. A quantitative estimate of the impact of both effects can be found in the ESI. $\dagger$

As far as the shapes of the XP spectra are concerned, they agree very well between the simulations and experiments. This can be seen in Fig. 5, where it should be noted that the energy scale in the simulations has been shifted to higher BEs by $18.95 \mathrm{eV}$. This value has been chosen to make the calculated and experimental spectra of PPz/Au lie on top of each other and, for the sake of consistency, was then also chosen in the PPd case. Such a shift is necessary considering that within the initial state approach one approximates the ionization energies by the (Kohn-Sham) orbital energies of the core levels. In this way, one only obtains core-level shifts, which eventually need to be aligned relative to a reference system. ${ }^{90-93}$

For $\mathrm{PPz} / \mathrm{Au}$, the simulations fully reproduce the occurrence of a pronounced shoulder at higher binding energies (the feature denoted as " 1 " in the XP spectrum of PPz/Au in Fig. 2b). 


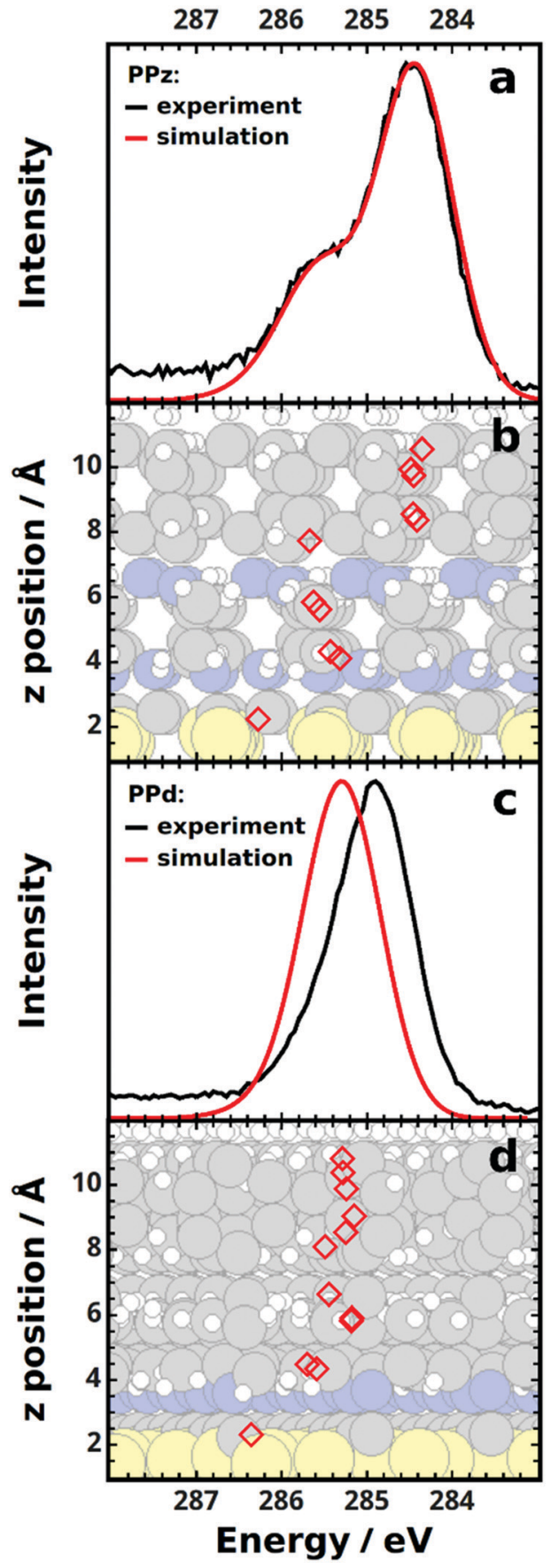

Fig. 5 Comparison between experimental and suitably aligned (see below) simulated C 1s XP spectra. (a) Experimental (black) and simulated (red) C 1s spectra of PPz/Au(111); (b) calculated core level energies for each carbon atom in the PPz molecule in the respective SAM including screening by the metal; (c) experimental (black) and simulated (red) C 1s XP spectra of PPd/Au(111); and (d) calculated core level energies for each carbon atom in the PPd molecule in the respective SAM including screening by the metal. Please note that in panels $b$ and $d$ the energy values are given for only one of the two molecules in the full coverage unit cell. Since these two molecules are differently tilted, they are not symmetry equivalent and the calculated energy values vary slightly between them. Such differences are, however, very small, as shown in the ESI. $\dagger$ To ease the comparison between simulations and experiments, the energy scale for the simulated data has been shifted to higher BEs by $18.95 \mathrm{eV}$ (aligning the measured and calculated spectra for PPz).
Conversely, for PPd/Au only a single dominant peak is observed in full agreement with the experiments. The atomically resolved core-level binding energies in Fig. $5 \mathrm{~b}$ and $\mathrm{d}$ support the original peak assignment from Section 3.1; i.e. the low $\mathrm{BE}$ feature in $\mathrm{PPz} /$ $\mathrm{Au}$ is associated with the carbon atoms in positions $2-6$ of the topmost phenyl ring, while the weaker, high $\mathrm{BE}$ feature originates from the carbon atoms directly bonded to the nitrogen atoms in the piperazine moiety. Conversely, for PPd/Au the core-level BEs of all C atoms in the rings are between 285.3 and $285.7 \mathrm{eV}$; as a consequence of this comparably small energetic spread and due to the absence of two energetically distinct groups of carbon atoms, we obtain only a somewhat broadened single peak that dominates the spectrum.

Beyond confirming and refining the peak assignment, the simulations provide additional insight that is not apparent in the experiments due to the finite energy resolution: (i) they reveal that in $\mathrm{PPz} / \mathrm{Au}$ the core level energy of the topmost carbon in the phenyl ring is shifted by $0.2 \mathrm{eV}$ compared to the neighboring carbons, an effect that is not observed in PPd/Au. (ii) The aliphatic carbons in PPd/Au have essentially the same BEs as the aromatic ones in the topmost ring. (iii) Finally, the $\mathrm{BE}$ associated with the carbon atom bonded to the two sulfurs is shifted to higher BEs by $\sim 1.0 \mathrm{eV}(1.3 \mathrm{eV})$ in PPz/Au and by $\sim 0.7 \mathrm{eV}(1.0 \mathrm{eV})$ in PPd/Au compared to the $\mathrm{C}$ atoms in the lower ring, disregarding (including) screening effects. Due to the finite escape depth of the photoelectrons, this is at best manifested as a high BE tail in the experimental spectra.

To understand the above-described peculiarities of the XP spectra, it is useful to keep in mind that core-level energies are determined by two factors: the local chemical environment of an atom and the local electrostatic energy. ${ }^{20,21,40,60,94}$ The latter can, for example, be changed through collective electrostatic effects via a periodic arrangement of polar groups common in $\mathrm{SAMs}^{21}$ or by the Madelung energy in ionic crystals. ${ }^{95,96}$ Conceptually, chemically and electrostatically induced shifts can be separated by reducing the density of the polar moieties in the adsorbate layer. ${ }^{21}$ This is straightforward, at least as long as reducing the dipole density does not modify atomic charges, e.g., by changing the charge transfer between the substrate and adsorbate. For atoms in a SAM that are at a sufficient distance from the docking groups, this means that core-level energies will depend on the coverage only, if there are dipole layers between them and the metal substrate. ${ }^{21}$ To analyze this, one needs to compare the core levels of densely packed films to those obtained from a calculation carried out at significantly reduced molecular coverage. In the latter case, the remaining molecules need to be fixed in the geometries they adopted in the dense SAM in order to avoid a superposition of effects arising from molecular rearrangements (see Section 2.3).

When applying this approach to the PPd and PPz SAMs, the spectra shown in Fig. 6 are obtained for full, 1/16 and 1/36 coverage. Their comparison reveals three crucial aspects: (i) the low-coverage spectra are significantly shifted with respect to the full coverage ones with the shifts of the $1 / 36$ spectra slightly exceeding those of the $1 / 16$ spectra; (ii) the shape of the spectra remains essentially unchanged; i.e., there is a rigid shift; and 
(iii) the shift for the PPd SAM (1.90 eV between full and 1/36 coverage, as measured from the positions of the maxima) clearly exceeds that for the PPz SAM $(1.32 \mathrm{eV})$. As can be inferred from the description in the preceding paragraph, the first aspect suggests that significant dipoles are present in the system; the second indicates that the most significant dipoles are in the region of the immediate metal/SAM interface, i.e., closer to the metal than the carbon atoms most strongly contributing to the $\mathrm{C} 1 \mathrm{~s}$ spectra; finally, the third aspect implies that this dipole is larger for PPd than for PPz. In addition, the comparably small shift between the 1/16 and 1/36 spectra for both PPd and PPz SAMs suggests a convergence of the calculations in terms of approaching the isolated-molecule limit. We note that the pronounced difference in the core level shifts observed for $\mathrm{PPz}$ and $\mathrm{PPd}$ in Fig. 6 cannot result from a different tilt angle of the DTC anchoring group. According to our calculations, the plane spanned by the $\mathrm{S}-\mathrm{S}-\mathrm{N}$ atoms (with $\mathrm{N}$ being the nitrogen atom that belongs to the DTC group) forms an angle of $6^{\circ}$ and $5.8^{\circ}$ relative to the surface normal for PPz and PPd, respectively, which is a very small difference and, thus, cannot significantly affect the interface dipole.

To consider the above trends in more detail, we analyze the atomistically resolved core-level energies shown in Fig. 7 for the PPd and PPz SAMs at full and 1/36 coverage. In this context we report the as calculated core-level energies; i.e., no rigid shift of the spectra is applied and no screening of the core hole by the metal substrate is considered. Otherwise, "artificial" shifts due to different dielectric constants of the adsorbate layer at

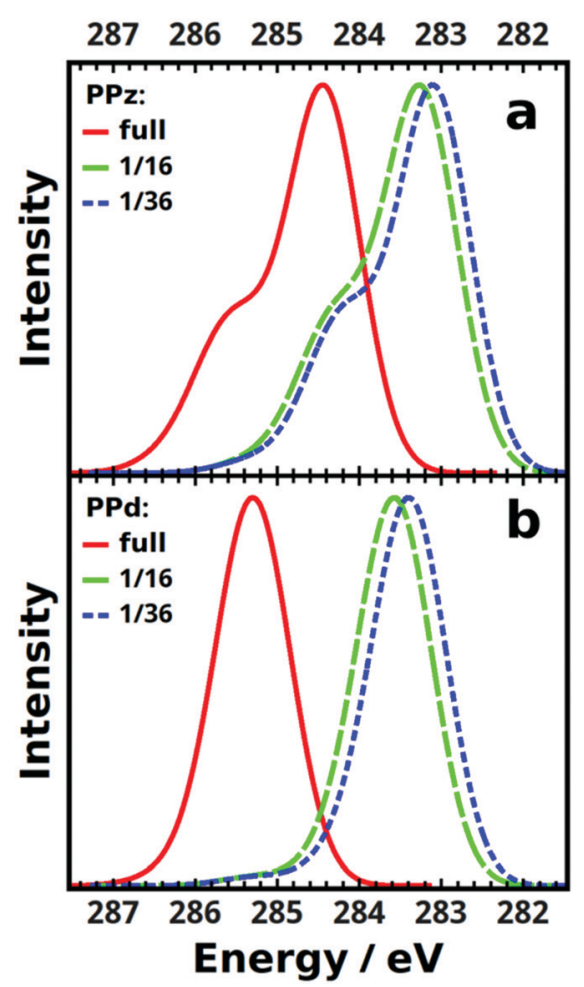

Fig. 6 Comparison between the simulated XP spectra of PPz (a) and PPd (b) on Au(111) obtained for full, $1 / 16$ and $1 / 36$ coverage. The legend is given in the panels. different coverages ( $c f$., eqn (1)) would obscure the more fundamental trends.

We start with a discussion of the $\mathrm{PPz}$ case (Fig. 7a): the horizontal arrows associated with the $\mathrm{C}$ atoms most strongly contributing to the XP spectrum have very similar lengths (average $\Delta E_{\mathrm{BE}} \approx 1.15 \mathrm{eV}$, for numerical details see ESI $\dagger$ ). This supports the conclusion drawn already from the rigid shift of the spectra that significant dipoles exist that are mostly found in the vicinity of the docking region.

Interestingly, $\Delta E_{\mathrm{BE}}$ is essentially the same also for the topmost $\mathrm{C}$ atom, which suggests a chemical origin for the different binding energy of that atom compared to the other phenyl carbons. As this difference is not observed in the PPd case (Fig. 7b), its origin must be the $\mathrm{N}$ atom bonded to the $\mathrm{C}$ in the 1-position of the phenyl ring in PPz. Its impact can be more straightforwardly investigated using aniline as a model system. Considering the possible resonance structures of aniline indeed allows rationalizing the chemical origin of the shift, as described in detail in the ESI. $\dagger$

For the carbon atoms in positions 3 and 5 and in positions 2 and 6 of the piperazine ring, the shift is on average reduced to $\sim 1.05 \mathrm{eV}$ and $\sim 0.72 \mathrm{eV}$, respectively, and it almost disappears $\left(\Delta E_{\mathrm{BE}}=0.12 \mathrm{eV}\right)$ for the carbon atom bonded to the two sulfurs (see Fig. 7a). The shift of the 1s energies of the lower nitrogen between full $(378.80 \mathrm{eV})$ and $1 / 36$ coverage $(378.39 \mathrm{eV})$ amounts to $0.41 \mathrm{eV}$, which is intermediate between that for the two adjacent $\mathrm{C}$ atoms. This, together with the vanishingly small

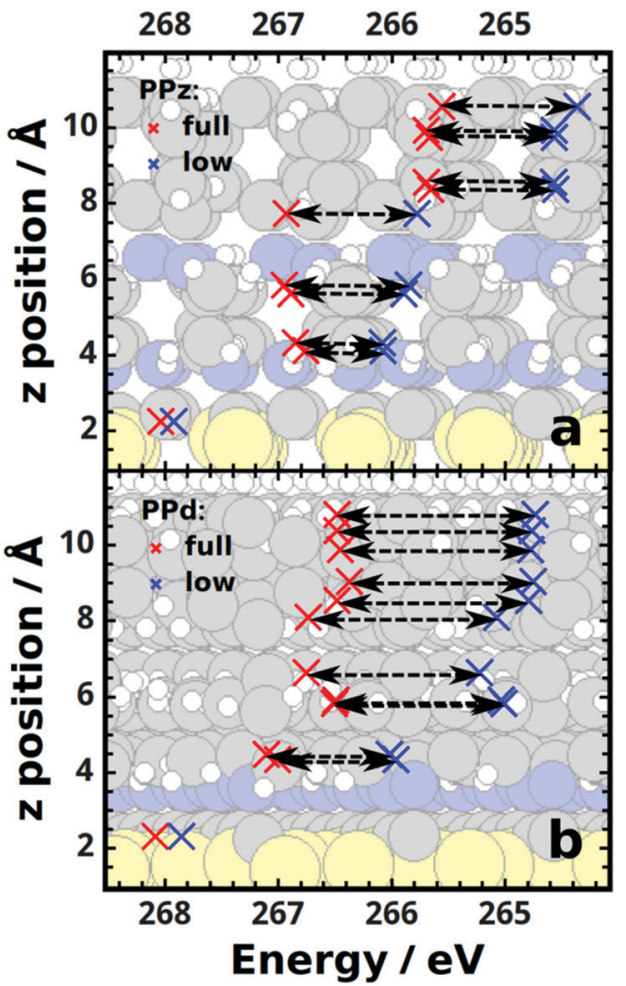

Fig. 7 Calculated, atomically resolved $C$ 1s core-level energies for the PPz (a) and PPd (b) SAMs obtained for full (red crosses) and low (1/36; blue crosses) coverage. The black dashed arrows serve as guides to the eye. 
coverage-dependent shift of the $S 2 p$ core levels energies (calculated to be $0.045 \mathrm{eV}$ on average), suggests that the dipoles causing the electrostatic shifts reside in the region around those atoms.

Here, it, however, has to be noted that an analysis of the coverage-dependence of the core-level shifts of atoms close to the substrate and in the immediate vicinity of the dipole is not straightforward. The reasons for that are two-fold: on the one hand, coverage dependent interfacial charge rearrangements resulting from the formation of the metal to SAM bond ${ }^{97}$ lead to coverage-dependent chemical shifts, which can easily mask the electrostatic ones. This aspect is discussed in detail in the ESI. $\dagger$ On the other hand, for reduced dipole densities the change in electrostatic energy close to the dipoles can no longer be approximated by a simple step. ${ }^{65}$ Consequently, at low coverages and close to the dipoles the magnitudes of electrostatic core-level shifts subtly depend on the exact positions of the respective atoms.

In passing we note that in an attempt to pinpoint the exact location of the dipole, we also tested various charge partitioning schemes, but (not really unexpectedly) observed significant variations between different SAM conformations and methodological approaches rendering these attempts inconclusive.

With regards to the coverage-dependent core-level energies of $\mathrm{PPd} / \mathrm{Au}$, qualitatively similar trends as in $\mathrm{PPz} / \mathrm{Au}$ are observed (see Fig. 7b and ESI $\dagger$ ), the main difference being that the overall coverage-dependent shift is larger even for the bottom ring, indicative of a larger dipole in the region of the docking group.

3.4.3. Docking positions and their impact on $S$ core-level binding energies. As indicated already in the discussion of the experimental results, we find docking positions of the four $S$ atoms per unit cell varying between on-top and bridge, including also displacements towards the hollow sites (for a plot of the S docking positions in the lowest energy structures see ESI $\dagger$ ). This results in calculated $S 2 p$ binding energies varying over $c a$. $0.13 \mathrm{eV}$ for $\mathrm{PPz} / \mathrm{Au}$ and $0.12 \mathrm{eV}$ for $\mathrm{PPd} / \mathrm{Au}$, which is consistent with the comparably large fwhm of the experimental S 2p XP spectra (see Section 3.1).

3.4.4. Work-function modifications. As far as the work function modifications (see Table 3) are concerned, the value obtained for the PPz SAM somewhat overestimates the measured one $(-1.7 \mathrm{eV}$ vs. $-1.3 \mathrm{eV})$. In fact, already in the past we have made similar observations, with one of the reasons being finite grain sizes and a correspondingly inevitable disorder in the real SAMs. ${ }^{20}$

As a matter of curiosity, it should be mentioned that in the PPz SAMs changing the molecular conformation by rotating the phenyl ring such that it is nearly perpendicular to the piperazine unit (twisted configuration) massively increases the work function change. This can be traced back to a significant increase of the dipole moment of the molecule (for an isolated molecule it increases from 2.8 Debye for the planar configuration to 3.9 Debye for the twisted one). Even though in the experiments there is no indication of the occurrence of the twisted structure, this result shows that inducing a twist (e.g., by chemical substitutions) could be a viable strategy for boosting the molecular dipole moment of $\mathrm{PPz}$ derivatives and to maximize the achievable work-function modification. Note that for the PPd SAM, a change from the twisted to the planar configuration has a much weaker impact on the molecular dipoles and on the work function changes (see Table 3 and ESI $\dagger$ ), which we attribute to the absence of heteroatoms in the vicinity of the rotated bond.

When comparing the calculated SAM-induced work function decrease of $\mathrm{PPz}$ and PPd, PPd yields a work function that is larger by $0.87 \mathrm{eV}$. This coincides with the shifts in the simulated core-level binding energies for $\mathrm{C}$ 1s states in the phenyl ring (see the last two columns of Table 3), a correlation that is expected based on the equivalent electrostatic origins of corelevel shifts and work function changes (see above and ref. 21). In the experiments, however, a distinctly smaller difference in the work functions of PPz and PPd samples is observed, which is on the order of only $0.2 \mathrm{eV}$. This is even smaller than the measured $0.42 \mathrm{eV}$ shift in the binding energy of the main features in the XP spectra related to the phenyl C 1s signal. The origin of this deviation between theory and experiment is not fully understood, and appears too large to be solely attributed to the lower coverage of the PPd SAMs as reported in Table 1 . Here, we cannot fully exclude a certain parameter variation for the different samples employed for the different measurements within the project. Also, we cannot rule out an overestimation of the particularly large calculated dipole associated with the bonded DTC group for the PPd system.

\section{Summary}

In the present work we combined synchrotron-based XPS, NEXAFS spectroscopy, work function measurements and state-of-the-art DFT calculations to study the molecular organization and electrostatic properties of SAMs on $\mathrm{Au}(111)$ formed by dithiocarbamates with rod-like symmetry, taking several representative monolayers as test systems. As the piperazine/ piperidine unit constitutes a common platform of these derivatives, particular emphasis is given to the investigation of the two parent compounds, PPd and PPz. They can be substituted with different functional terminal groups, thus allowing a wide range of molecular dipoles and offering a promising avenue for work function engineering at metal-organic interfaces. From our study, it emerges that the DTC anchoring group represents a useful building block for monomolecular self-assembly on coinage metal substrates. Its combination with the piperidine or piperazine moiety allows for a sufficient flexibility, enabling the self-assembly of more rigid terminal groups (such as the phenyl rings in the case of PPd, PPz, and FP-Pz). It also allows the substitution with additional groups such as secondary amines, alkoxy groups, cyano groups, and many more (e.g. for $\mathrm{C} 12 \mathrm{~N}-\mathrm{PPd}$ and OMe-PPz). Importantly, both sulfur atoms in the DTC anchoring group bind to the substrate in a bidentate fashion, a very advantageous feature distinguishing the DTC moiety from other thiol-based dipodal and tripodal docking groups, which frequently show a heterogeneous chemistry for 
each individual sulfur atom (with coexisting thiolate, atomic sulfur, unbound sulfur, and disulfide configurations). Notwithstanding its bulkier bonding configuration, the DTC anchor, in combination with the piperidine or piperazine linkers, allows a dense molecular packing (see Table 1), with almost the same density as for monodentate docking groups such as thiolates in alkanethiolate and arylthiolate SAMs on $\mathrm{Au}(111)$. Such a dense molecular packing is characterized by small molecular inclinations, as evidenced both by NEXAFS experiments and theoretical simulations (see Tables 2 and 3). This inclination is only slightly altered upon substitution of the PPd and PPz backbones with dipolar tail groups, or by partial fluorination of these moieties, a very favorable property in view of interfacedipole engineering, e.g. for the efficient alignment of transport levels in organic semiconductors.

As an overall trend, we find excellent agreement between the results from spectroscopic experiments and theoretical simulations in terms of molecular organization of PPd and PPz films. This not only refers to the molecular orientation but, for example, also to the heterogeneity of sulfur absorption sites in DTC anchoring groups. In addition, the XP spectra of the films could be reproduced by theory, in spite of a certain deviation between calculated and measured work function values. The most important conclusion with regards to the electrostatic properties of PPd and PPz SAMs concerns the location of the electrostatic dipole layer within these films, found to be in a region immediately adjacent to the metal/SAM interface. Interestingly, there is no significant potential step in the region of the additional nitrogen atom in $\mathrm{PPz}$, at least as long as in $\mathrm{PPz}$ a coplanar molecular geometry prevails. The additional nitrogen atoms, however, induce a minor chemical shift affecting the carbon atoms in the terminal phenyl ring in the PPz SAM.

The most relevant difference found between the PPd and PPz platforms is a more upright orientation of the molecular backbone associated with a higher coverage for $\mathrm{PPz}$, making especially this platform promising in view of further substitution of the terminal phenyl ring by polar groups, thereby providing access to a broad variety of interface dipoles.

\section{Conflicts of interest}

There are no conflicts to declare.

\section{Acknowledgements}

The authors thank the Max IV facility and the HelmholtzZentrum Berlin (BESSY II) for the allocation of the beamtime, A. Nefedov and Ch. Wöll for technical cooperation at BESSY II, A. Preobrajenski for valuable technical assistance at Max IV, and BESSY II and Max IV staff for the technical support. We also thank Oliver T. Hofmann and Thomas C. Taucher for fruitful discussions regarding the computational part of this study. This work has been supported financially by the German Research Foundation (DFG; grant ZH 63/22-1) and by the
Austrian Science Fund (FWF): I2081-N20. The computational results presented have been achieved using the Vienna Scientific Cluster (VSC).

\section{References}

1 I. H. Campbell, S. Rubin, T. A. Zawodzinski, J. D. Kress, R. L. Martin, D. L. Smith, N. N. Barashkov and J. P. Ferraris, Phys. Rev. B: Condens. Matter Mater. Phys., 1996, 54, 14321-14324.

2 B. de Boer, A. Hadipour, M. M. Mandoc, T. van Woudenbergh and P. W. M. Blom, Adv. Mater., 2005, 17, 621-625.

3 G. Heimel, L. Romaner, J. L. Brédas and E. Zojer, Phys. Rev. Lett., 2006, 96, 196806.

4 B. H. Hamadani, D. A. Corley, J. W. Ciszek, J. M. Tour and D. Natelson, Nano Lett., 2006, 6, 1303-1306.

5 X. Cheng, Y.-Y. Noh, J. Wang, M. Tello, J. Frisch, R.-P. Blum, A. Vollmer, J. P. Rabe, N. Koch and H. Sirringhaus, Adv. Funct. Mater., 2009, 19, 2407-2415.

6 S. A. DiBenedetto, A. Facchetti, M. A. Ratner and T. J. Marks, Adv. Mater., 2009, 21, 1407-1433.

7 D. Boudinet, M. Benwadih, Y. Qi, S. Altazin, J.-M. Verilhac, M. Kroger, C. Serbutoviez, R. Gwoziecki, R. Coppard, G. Le Blevennec, A. Kahn and G. Horowitz, Org. Electron., 2010, 11, 227-237.

8 M. Halik and A. Hirsch, Adv. Mater., 2011, 23, 2689-2695.

9 J. M. Chiu and Y. Tai, ACS Appl. Mater. Interfaces, 2013, 5, 6946-6950.

10 I. Lange, S. Reiter, M. Pätzel, A. Zykov, A. Nefedov, J. Hildebrandt, S. Hecht, S. Kowarik, C. Wöll, G. Heimel and D. Neher, Adv. Funct. Mater., 2014, 24, 7014-7024.

11 C. K. Song, K. A. Luck, N. Zhou, L. Zeng, H. M. Heitzer, E. F. Manley, S. Goldman, L. X. Chen, M. A. Ratner, M. J. Bedzyk, R. P. H. Chang, M. C. Hersam and T. J. Marks, J. Am. Chem. Soc., 2014, 136, 17762-17773.

12 J. Kim, Y. S. Rim, Y. Liu, A. C. Serino, J. C. Thomas, H. Chen, Y. Yang and P. S. Weiss, Nano Lett., 2014, 14, 2946-2951.

13 F. Piersimoni, R. Schlesinger, J. Benduhn, D. Spoltore, S. Reiter, I. Lange, N. Koch, K. Vandewal and D. Neher, J. Phys. Chem. Lett., 2015, 6, 500-504.

14 H. Ju Lee, A. C. Jamison and T. R. Lee, Acc. Chem. Res., 2015, 48, 3007-3015.

15 S. Casalini, C. A. Bortolotti, F. Leonardi and F. Biscarini, Chem. Soc. Rev., 2017, 46, 40-71.

16 A. Petritz, M. Krammer, E. Sauter, M. Gärtner, G. Nascimbeni, B. Schrode, A. Fian, H. Gold, A. Cojocaru, E. Karner-Petritz, R. Resel, A. Terfort, E. Zojer, M. Zharnikov, K. Zojer and B. Stadlober, Adv. Funct. Mater., 2018, 28, 1804462.

17 A. Ulman, Chem. Rev., 1996, 96, 1533-1554.

18 J. C. Love, L. A. Estroff, J. K. Kriebel, R. G. Nuzzo and G. M. Whitesides, Chem. Rev., 2005, 105, 1103-1169.

19 C. Schmidt, A. Witt and G. Witte, J. Phys. Chem. A, 2011, 115, 7234-7241.

20 T. Abu-Husein, S. Schuster, D. A. Egger, M. Kind, T. Santowski, A. Wiesner, R. Chiechi, E. Zojer, A. Terfort and M. Zharnikov, Adv. Funct. Mater., 2015, 25, 3943-3957. 
21 T. C. Taucher, I. Hehn, O. T. Hofmann, M. Zharnikov and E. Zojer, J. Phys. Chem. C, 2016, 120, 3428-3437.

22 O. M. Cabarcos, S. Schuster, I. Hehn, P. P. Zhang, M. M. Maitani, N. Sullivan, J.-B. Giguère, J.-F. Morin, P. S. Weiss, E. Zojer, E. Zojer, M. Zharnikov and D. L. Allara, J. Phys. Chem. C, 2017, 121, 15815-15830.

23 E. Sauter, C.-O. Gilbert, J. Boismenu-Lavoie, J.-F. Morin and M. Zharnikov, J. Phys. Chem. C, 2017, 121, 23017-23024.

24 M. Gärtner, E. Sauter, G. Nascimbeni, A. Petritz, A. Wiesner, M. Kind, T. Abu-Husein, M. Bolte, B. Stadlober, E. Zojer, A. Terfort and M. Zharnikov, J. Phys. Chem. C, 2018, 122, 28757-28774.

25 S. A. Paniagua, A. J. Giordano, O. N. L. Smith, S. Barlow, H. Li, N. R. Armstrong, J. E. Pemberton, J.-L. Brédas, D. Ginger and S. R. Marder, Chem. Rev., 2016, 116, 7117-7158.

26 K. M. Knesting, P. J. Hotchkiss, B. A. MacLeod, S. R. Marder and D. S. Ginger, Adv. Mater., 2012, 24, 642-646.

27 F. von Wrochem, D. Gao, F. Scholz, H. G. Nothofer, G. Nelles and J. M. Wessels, Nat. Nanotechnol., 2010, 5, 618-624.

28 J. M. Wessels, H. G. Nothofer, W. E. Ford, F. von Wrochem, F. Scholz, T. Vossmeyer, A. Schroedter, H. Weller and A. Yasuda, J. Am. Chem. Soc., 2004, 126, 3349-3356.

29 Y. Zhao, W. Pérez-Segarra, Q. Shi and A. Wei, J. Am. Chem. Soc., 2005, 127, 7328-7329.

30 D. Gao, F. Scholz, H.-G. Nothofer, W. E. Ford, U. Scherf, J. M. Wessels, A. Yasuda and F. von Wrochem, J. Am. Chem. Soc., 2011, 133, 5921-5930.

31 N. E. A. Reeler, K. A. Lerstrup, W. Somerville, J. Speder, S. V. Petersen, Bo W. Laursen, M. Arenz, X. Qiu, T. Vosch and K. Nørgaard, Sci. Rep., 2015, 5, 15273.

32 J. B. Simonsen, N. E. A. Reeler, A. Fossum, K. A. Lerstrup, Bo W. Laursen and K. Nørgaard, Nano Res., 2016, 9, 3093-3098.

33 W. E. Ford, D. Gao, N. Knorr, R. Wirtz, F. Scholz, Z. Karipidou, K. Ogasawara, S. Rosselli, V. Rodin, G. Nelles and F. von Wrochem, ACS Nano, 2014, 8, 9173-9180.

34 D. Meyer, T. Schäfer, P. Schulz, S. Jung, J. Rittich, D. Mokros, I. Segger, F. Maercks, C. Effertz, R. Mazzarello and M. Wuttig, Langmuir, 2016, 32, 8812-8817.

35 Y. Zhao, J. N. Newton, J. Liu and A. Wei, Langmuir, 2009, 25, 13833-13839.

36 W. E. Ford, D. Gao, F. Scholz, G. Nelles and F. von Wrochem, ACS Nano, 2013, 7, 1943-1951.

37 R. Naumann, S. M. Schiller, F. Giess, B. Grohe, K. B. Hartman, I. Karcher, I. Koper, J. Lubben, K. Vasilev and W. Knoll, Langmuir, 2003, 19, 5435-5443.

38 S. Nakamaru, F. Scholz, W. E. Ford, Y. Goto and F. von Wrochem, Adv. Mater., 2017, 29, 1605924.

39 F. Chesneau, J. Zhao, C. Shen, M. Buck and M. Zharnikov, J. Phys. Chem. C, 2010, 114, 7112-7119.

40 I. Hehn, S. Schuster, T. Wächter, T. Abu-Husein, A. Terfort, M. Zharnikov and E. Zojer, J. Phys. Chem. Lett., 2016, 7, 2994-3000.

41 J. Stöhr, NEXAFS spectroscopy, Springer-Verlag, Berlin, 1992. 42 M. Zharnikov, J. Electron Spectr. Relat. Phenom., 2010, 178179, 380-393.
43 J. F. Moulder, W. E. Stickle, P. E. Sobol and K. D. Bomben, Handbook of X-ray Photoelectron Spectroscopy, ed. J. Chastian, PerkinElmer Corp., Eden Prairie, MN, 1992.

44 J. Thome, M. Himmelhaus, M. Zharnikov and M. Grunze, Langmuir, 1998, 14, 7435-7449.

45 F. Chesneau, B. Schüpbach, K. Szelagowska-Kunstman, N. Ballav, P. Cyganik, A. Terfort and M. Zharnikov, Phys. Chem. Chem. Phys., 2010, 12, 12123-12127.

46 M. Ratner and D. Castner, in Surface Analysis - The principal techniques, ed. J. Vickerman, Wiley, Chichester, 1997.

47 C. L. A. Lamont and J. Wilkes, Langmuir, 1999, 15, 2037-2042.

48 F. Schreiber, Prog. Surf. Sci., 2000, 65, 151-256.

49 M. Zharnikov and M. Grunze, J. Phys.: Condens. Matter., 2001, 13, 11333-11365.

50 P. E. Batson, Phys. Rev. B: Condens. Matter Mater. Phys., 1993, 48, 2608-2610.

51 V. Blum, R. Gehrke, F. Hanke, P. Havu, V. Havu, X. Ren, K. Reuter and M. Scheffler, Comput. Phys. Commun., 2009, 180, 2175-2196.

52 J. P. Perdew, K. Burke and M. Ernzerhof, Phys. Rev. Lett., 1996, 77, 3865-3868; Erratum: Phys. Rev. Lett., 1997, 78, 1396.

53 A. Tkatchenko and M. Scheffler, Phys. Rev. Lett., 2009, 102, 073005.

54 V. G. Ruiz, W. Liu, E. Zojer, M. Scheffler and A. Tkatchenko, Phys. Rev. Lett., 2012, 108, 146103.

55 C. Freysoldt, P. Eggert, P. Rinke, A. Schindlmayr and M. Scheffler, Phys. Rev. B: Condens. Matter Mater. Phys., 2008, 77, 214517.

56 E. Verwüster, O. T. Hofmann, D. A. Egger and E. Zojer, J. Phys. Chem. C, 2015, 119, 7817-7825.

57 E. Pehlke and M. Scheffler, Phys. Rev. Lett., 1993, 71, 2338-2341.

58 S. Lizzit, S. Baraldi, A. Groso, K. Reuter, M. V. GandugliaPirovano, C. Stampfl, M. Scheffler, M. Stichler, C. Keller, W. Wurth and D. Menzel, Phys. Rev. B: Condens. Matter Mater. Phys., 2001, 63, 205419.

59 W. Olovsson, C. Göransson, T. Marten and I. A. Abrokosov, Phys. Status Solidi B, 2006, 243, 2447-2464.

60 J. L. Cabellos, D. J. Mowbray, E. Goiri, A. El-Sayed, L. Floreano, D. G. de Oteyza, C. Rogero, J. E. Ortega and A. Rubio, J. Phys. Chem. C, 2012, 116, 17991-18001.

61 S. García-Gil, A. García and P. Ordejón, Eur. Phys. J. B, 2012, 85, 239.

62 E. J. Baerends, O. V. Gritsenko and R. van Meer, Phys. Chem. Chem. Phys., 2013, 16, 16408-16425.

63 N. Pueyo Bellafont, F. Illas and P. S. Bagus, Phys. Chem. Chem. Phys., 2015, 17, 4015-4019.

64 N. Pueyo Bellafont, P. S. Bagus and F. Illas, J. Chem. Phys., 2015, 142, 214102.

65 G. Heimel, F. Rissner and E. Zojer, Adv. Mater., 2010, 22, 2494-2513.

66 J. D. Jackson, Classical electrodynamics, Wiley, New York, 3rd edn, 1999.

67 J. Neaton, M. Hybertsen and S. Louie, Phys. Rev. Lett., 2006, 97, 216405. 
68 Y. Li, D. Lu and G. Galli, J. Chem. Theory Comput., 2009, 5, 881-886.

69 D. A. Egger, Z.-F. Liu, J. B. Neaton and L. Kronik, Nano Lett., 2015, 15, 2448-2455.

70 O. M. Cabarcos, A. Shaporenko, T. Weidner, S. Uppili, L. S. Dake, M. Zharnikov and D. L. Allara, J. Phys. Chem. C, 2008, 112, 10842-10854.

71 J.-S. Park, A. N. Vo, D. Barriet, Y. S. Shon and T. R. Lee, Langmuir, 2005, 21, 2902-2911.

72 T. Weidner, N. Ballav, U. Siemeling, D. Troegel, T. Walter, R. Tacke, D. G. Castner and M. Zharnikov, J. Phys. Chem. C, 2009, 113, 19609-19617.

73 F. Sander, T. Peterle, N. Ballav, F. von Wrochem, M. Zharnikov and M. Mayor, J. Phys. Chem. C, 2010, 114, 4118-4125.

74 M. Lindner, M. Valášek, J. Homberg, K. Edelmann, L. Gerhard, W. Wulfhekel, O. Fuhr, T. Wächter, M. Zharnikov, V. Kolivoška, L. Pospisil, G. Meszaros, M. Hromadova and M. Mayor, Chem. Eur. J., 2016, 22, 13218-13235.

75 W. E. Ford, F. Abraham, F. Scholz, G. Nelles, G. Sandford and F. von Wrochem, J. Phys. Chem. C, 2017, 121, 1690-1703.

76 S. Frey, A. Shaporenko, M. Zharnikov, P. Harder and D. L. Allara, J. Phys. Chem. B, 2003, 107, 7716-7725.

77 A. Shaporenko, A. Terfort, M. Grunze and M. Zharnikov, J. Electron Spectrosc. Relat. Phenom., 2006, 151, 45-51.

78 A. P. Hitchcock, P. Fisher, A. Gedanken and M. B. Robin, J. Phys. Chem., 1987, 91, 531-540.

79 T. Yokoyama, K. Seki, I. Morisada, K. Edamatsu and T. Ohta, Phys. Scr., 1990, 41, 189-192.

80 S. Frey, V. Stadler, K. Heister, W. Eck, M. Zharnikov, M. Grunze, B. Zeysing and A. Terfort, Langmuir, 2001, 17, 2408-2415.

81 O. Plashkevych, A. Snis, L. Yang, H. Ågren and S. F. Matar, Phys. Scr., 2001, 63, 70-86.
82 Y. Zubavichus, M. Zharnikov, A. Shaporenko and M. Grunze, J. Electron Spectrosc. Relat. Phenom., 2004, 134, 25-33.

83 P. S. Bagus, K. Weiss, A. Schertel, Ch. Wöll, W. Braun, H. Hellwig and C. Jung, Chem. Phys. Lett., 1996, 248, 129.

84 O. Plashkevych, L. Yang, O. Vahtras, H. Agren and L. G. M. Pettersson, Chem. Phys., 1997, 222, 125-137.

85 N. Ballav, B. Schüpbach, O. Dethloff, P. Feulner, A. Terfort and M. Zharnikov, J. Am. Chem. Soc., 2007, 129, 15416-15417.

86 Z. Zhang, T. Wächter, M. Kind, S. Schuster, J. W. Bats, A. Nefedov, M. Zharnikov and A. Terfort, ACS Appl. Mater. Interfaces, 2016, 8, 7308-7319.

87 W. Azzam, C. Fuxen, A. Birkner, H.-T. Rong, M. Buck and C. Wöll, Langmuir, 2003, 19, 4958-4968.

88 A. Bashir, E. Sauter, M. Rohwerder, M. Zharnikov and W. Azzam, ChemPhysChem, 2017, 18, 702-714.

89 E. Verwüster, E. Wruss, E. Zojer and O. T. Hofmann, J. Chem. Phys., 2017, 147, 024706.

90 J. Vackár̆, M. Hyt'ha and A. Šimůnek, Phys. Rev. B: Condens. Matter Mater. Phys., 1998, 58, 12712-12720.

91 Y. Morikawa, T. Hayashi, C. C. Liew and H. Nozoye, Surf. Sci., 2002, 507, 46-50.

92 G. Heimel, L. Romaner, J.-L. Brédas and E. Zojer, Surf. Sci., 2006, 600, 4548-4562.

93 M. Giesbers, A. T. M. Marcelis and H. Zuilhof, Langmuir, 2013, 29, 4782-4788.

94 A. El-Sayed, P. Borghetti, E. Goiri, C. Rogero, L. Floreano, G. Lovat, D. J. Mowbray, J. L. Cabellos, Y. Wakayama, A. Rubio, J. E. Ortega and D. G. de Oteyza, ACS Nano, 2013, 7, 6914-6920.

95 P. S. Bagus, G. Pacchioni, C. Sousa, T. Minerva and F. Parmigiani, Chem. Phys. Lett., 1992, 196, 641-646.

96 P. S. Bagus, E. S. Ilton and C. J. Nelin, Surf. Sci. Rep., 2013, 68, 273-304.

97 G. Heimel, L. Romaner, J.-L. Brédas and E. Zojer, Surf. Sci., 2006, 600, 4548-4562. 